\title{
Monitoring and modelling of white dwarfs with extremely weak magnetic fields
}

\section{WD 2047+372 and WD 2359-434^}

\author{
J. D. Landstreet ${ }^{1,2}$, S. Bagnulo ${ }^{1}$, G. Valyavin ${ }^{3}$, and A. F. Valeev ${ }^{3}$ \\ 1 Armagh Observatory and Planetarium, College Hill, Armagh BT61 9DG, UK \\ e-mail: jlandstr@uwo.ca \\ 2 Dept. of Physics \& Astronomy, University of Western Ontario, London, Ontario, Canada \\ ${ }^{3}$ Special Astrophysical Observatory, RAS, Nizhnij Arkhiz, Zelenchukskij Region, 369167 Karachai-Cherkessian Republic, Russia
}

Received 23 June 2017 / Accepted 28 August 2017

\begin{abstract}
Magnetic fields are detected in a few percent of white dwarfs. The number of such magnetic white dwarfs known is now some hundreds. Fields range in strength from a few $\mathrm{kG}$ to several hundred MG. Almost all the known magnetic white dwarfs have a mean field modulus $\geq 1 \mathrm{MG}$. We are trying to fill a major gap in observational knowledge at the low field limit $(\leq 200 \mathrm{kG})$ using circular spectro-polarimetry. In this paper we report the discovery and monitoring of strong, periodic magnetic variability in two previously discovered "super-weak field" magnetic white dwarfs, WD 2047+372 and WD 2359-434. WD 2047+372 has a mean longitudinal field that reverses between about -12 and $+15 \mathrm{kG}$, with a period of $0.243 \mathrm{~d}$, while its mean field modulus appears nearly constant at $60 \mathrm{kG}$. The observations can be interpreted in terms of a dipolar field tilted with respect to the stellar rotation axis. WD 2359-434 always shows a weak positive longitudinal field with values between about 0 and $+12 \mathrm{kG}$, varying only weakly with stellar rotation, while the mean field modulus varies between about 50 and $100 \mathrm{kG}$. The rotation period is found to be $0.112 \mathrm{~d}$ using the variable shape of the $\mathrm{H} \alpha$ line core, consistent with available photometry. The field of this star appears to be much more complex than a dipole, and is probably not axisymmetric. Available photometry shows that WD 2359-434 is a light variable with an amplitude of only 0.005 mag; our own photometry shows that if WD $2047+372$ is photometrically variable, the amplitude is below about $0.01 \mathrm{mag}$. These are the first models for magnetic white dwarfs with fields below about $100 \mathrm{kG}$ based on magnetic measurements through the full stellar rotation. They reveal two very different magnetic surface configurations, and that, contrary to simple ohmic decay theory, WD 2359-434 has a much more complex surface field than the much younger WD 2047+372.
\end{abstract}

Key words. white dwarfs - stars: magnetic field - stars: individual: WD 2047+372 - stars: individual: WD 2359-434

\section{Introduction}

It has been known since the 1970 s that a few percent of white dwarfs (WDs) have detectable surface magnetic fields (e.g. Kemp et al. 1970; Angel \& Landstreet 1971; Landstreet \& Angel 1971; Kawka et al. 2007). These fields are detected in some WDs by the presence of broad-band circular (and sometimes linear) polarisation, and in other stars by the observation of Zeeman splitting and circular polarisation in spectral lines such as the Balmer lines (e.g. Aznar Cuadrado et al. 2004; Kepler et al. 2013). The observed field strengths, based on measuring Zeeman splitting or polarisation, or on matching the positions of features in the spectrum,

\footnotetext{
* Based, in part, on observations collected at the European Organisation for Astronomical Research in the Southern Hemisphere, Chile, under observing programmes 095.D-0264 and 097.D-0264, and obtained from the ESO/ST-ECF Science Archive Facility; in part, on observations made with the William Herschel Telescope, operated on the island of La Palma by the Isaac Newton Group in the Spanish Observatorio del Roque de los Muchachos of the Instituto de Astrofisica de Canarias: and in part on observations obtained at the Canada-France-Hawaii Telescope (CFHT) which is operated by the National Research Council of Canada, the Institut National des Sciences de l'Univers of the Centre National de la Recherche Scientifique of France, and the University of Hawaii.
}

range from tens of $\mathrm{kG}(1 \mathrm{Tesla}=10 \mathrm{kG})$ to almost $1000 \mathrm{MG}$ (e.g. Jordan 1992; Putney \& Jordan 1995; Külebi et al. 2009; Landstreet et al. 2012).

Using these methods, during the first thirty years after the discovery of the first magnetic fields in white dwarfs, new magnetic white dwarfs (MWDs) were discovered at a rate of about two per year. The situation changed qualitatively as a result of the Sloan Digital Sky Survey (SDSS), which has led to the identification of several hundred new MWDs. However, these two data sets have quite different properties. The early MWD discoveries are mostly found among the "bright" WDs, with magnitudes of $13<V<16$, and many have been observed at reasonably high $\mathrm{S} / \mathrm{N}$, often by spectropolarimetry (e.g. Schmidt \& Smith 1995). The fields found in such stars cover the full range of observed strengths, from a few tens of $\mathrm{kG}$ to about 1000 MG. In contrast, almost all the SDSS MWDs are in the range $16<V<20$, and the spectra available for these stars typically have $S / N \sim 15$ or 20 . Due to the low resolving power and low $\mathrm{S} / \mathrm{N}$ values, the SDSS data only permit the discovery of fields above a threshold of 1-2 MG, and field strengths can only be usefully estimated for the higher $\mathrm{S} / \mathrm{N}$ spectra. Thus the huge increase in the number of identified MWDs has only increased knowledge of the high-field region of the full MWD field strength distribution, and most of these new MWDs can only be studied further using the largest telescopes. 
In spite of the large sample of MWDs accumulated over almost $50 \mathrm{yr}$, two fundamental questions remain unanswered.

- We do not understand why strong magnetic fields are present in a small fraction of white dwarfs, but no detectable fields are found in the large majority.

- We do not yet know how the observed fields evolve as the host stars age.

In the absence of well-developed theoretical responses to these two questions, observations can contribute guidance and clues of two different kinds. First, studies of magnetic characteristics of large samples of MWDs should make it possible to observationally determine how the magnitudes of surface fields evolve with WD age, potentially as a function of WD mass, composition, and space velocity (or population). Such statistical studies might help to identify source populations of red giants and/or AGB stars for MWDs of various kinds (e.g. Valyavin 2015). Secondly, detailed studies of individual MWDs should provide complementary clues to the ones from statistical studies. An important kind of information that can emerge from studying individual stars is the determination of the surface field structure, and following individual studies, how the structures found depend on field strength, cooling age, and so on.

We are carrying out a large survey, together with detailed studies of individual MWDs, to address several aspects of the two basic questions listed above. One aspect in which we are particularly interested is the modelling of the surface magnetic field of the individual MWDs with the very weakest fields, to obtain empirical information on whether the field structures are the same as in MWDs with strong fields, and eventually also how these surface field structures evolve with WD age. In some strong-field MWDs the observed field is found to vary periodically with periods ranging from minutes to weeks (e.g. Kawka et al. 2007; Brinkworth et al. 2013; Ferrario et al. 2015), revealing the rotation period (and total angular momentum) of the underlying star. This situation allows much more reliable magnetic mapping than is possible if the observed field is measured only once or twice, or is unvarying, as the variations make available a variety of views of the field structure as the star rotates. Accordingly, we are attempting to determine rotation periods of the super-weak field MWDs, following up with detailed modelling where possible.

At present, no reasonably constrained surface field model is available for any MWD with a global field below about $300 \mathrm{kG}$. In this paper we report the discovery and analysis of the magnetic variability of WD 2047+372 and WD 2359-434, two stars whose fields are among the very weakest clearly detected WD magnetic fields. We then use the observed variations of various kinds of measurements of each star to obtain the first wellconstrained surface field models for two MWDs with fields below $100 \mathrm{kG}$.

\section{Our targets}

The main physical parameters of the two MWDs discussed in this paper are given in Table 1. In the following we provide some further details about them.

\subsection{WD $2047+372$}

The weak magnetic field of the DA 3.4 WD 2047+372= G 21036 was first discovered in October 2015 on the basis of a spectropolarimetric observation with the intermediate-resolution ISIS spectro-polarimeter on the William Herschel Telescope
Table 1. Physical parameters of white dwarfs discussed in this paper.

\begin{tabular}{lll}
\hline \hline Parameter (units) & WD 2047+372 & WD 2359-434 \\
\hline$m_{V}(\mathrm{mag})$ & 12.93 & 13.05 \\
$\pi(\mathrm{mas})$ & $57.87 \pm 0.69$ & $127.4 \pm 6.8$ \\
$D(\mathrm{pc})$ & $17.3 \pm 0.7$ & $7.85 \pm 0.40$ \\
$T_{\text {eff }}(\mathrm{K})$ & $14712 \pm 286$ & $8648 \pm 123$ \\
$\log \left(L / L_{\odot}\right)$ & -2.34 & -3.26 \\
$\log g\left(\mathrm{~cm} \mathrm{~s}^{-2}\right)$ & $8.31 \pm 0.04$ & $8.29 \pm 0.05$ \\
$M / M_{\odot}$ & $0.81 \pm 0.03$ & $0.78 \pm 0.03$ \\
Composition & $\mathrm{H}(\mathrm{DA})$ & $\mathrm{H}(\mathrm{DA})$ \\
Age $(\mathrm{Gyr})$ & 0.34 & 1.37 \\
\hline
\end{tabular}

Notes. Data taken from Giammichele et al. (2012).

(WHT), followed by a spectropolarimetric observation obtained with high-resolution spectropolarimeter ESPaDOnS on the Canada-France-Hawaii Telescope (CFHT). Both observations revealed clear splitting of the deep and sharp non-local thermodynamic equilibrium (non-LTE) line core of $\mathrm{H} \alpha$, and both provided marginal detection of Zeeman polarisation in the line wings of $\mathrm{H} \alpha$. In addition, weak magnetic splitting was observed in the ESPaDOnS spectrum of the core of the $\mathrm{H} \beta$ line (Landstreet et al. 2016).

The initial discovery spectra provided measurements of the mean field modulus (or mean surface field) averaged over the visible hemisphere $\langle|B|\rangle$ of about $57 \mathrm{kG}$, and values for the mean longitudinal field averaged over the visible hemisphere $\left\langle B_{z}\right\rangle$ of about $1 \mathrm{kG}$ (ISIS) and $6 \mathrm{kG}(\mathrm{ESPaDOnS})$. It is worth noting that this WD is quite bright, and that its spectrum has repeatedly been studied in some detail (e.g. Greenstein \& Liebert 1990; Gianninas et al. 2011; Giammichele et al. 2012). A sensitive low-resolution polarimetric study by Schmidt \& Smith (1995) did not detect the magnetic field. In the end, the superweak field (the third-smallest field securely detected in a WD) was discovered using a combination of particularly high $\mathrm{S} / \mathrm{N}$, high spectral resolution, and polarimetry.

\subsection{WD 2359-434}

The presence of a magnetic field in the bright, cool DA 5.8 WD 2359-434 = LAWD 96 was first suggested by Koester et al. (1998) from the unusually sharp and shallow core of $\mathrm{H} \alpha$ in a high-resolution spectrum of the star obtained with the European Southern Observatory (ESO) high-resolution UVES spectrograph on the Very Large Telescope (VLT) for the supernova progenitor (SPY) programme. The presence of a field was confirmed using low-resolution spectropolarimetry with the ESO FORS1 low-resolution spectropolarimeter by Aznar Cuadrado et al. (2004), who detected fields of $\left\langle B_{z}\right\rangle \approx+3$ and $+4 \mathrm{kG}$ at the 5-6 $\sigma$ level of significance on two different nights (we note that these data were re-reduced as described by Bagnulo et al. (2015), and the sign of the observations changed to conform to the usual convention). The value of $\langle|B|\rangle$ was subsequently estimated from the UVES spectra to be about $110 \mathrm{kG}$ (Koester et al. 2009). Like WD 2047+372, this bright WD had been studied extensively but was only recognised as magnetic from high-resolution spectroscopy.

\section{Observations}

The data used in this work are summarised in Table 2 and come from different sources. Some data sets come from our own 
Table 2. Summary of the measurements used in this work.

\begin{tabular}{lll}
\hline \hline & WD 2047+372 & WD 2359-434 \\
\hline $\begin{array}{l}\text { HR spectroscopy } \\
\text { LR spectropolarimetry }\end{array}$ & & $\begin{array}{l}\text { 2 UVES observations (Koester et al. 2009) } \\
\text { 2 FORS1 observations (Aznar Cuadrado et al. 2004) } \\
\text { 4 FORS2 observations (this work) }\end{array}$ \\
MR spectropolarimetry & $\begin{array}{l}\text { 1 ISIS observations (Landstreet et al. 2016) } \\
\text { 4 MSS observations (this work) }\end{array}$ & \\
HR spectropolarimetry & $\begin{array}{l}\text { 1 ESPaDOnS observation (Landstreet et al. 2016) } \\
\text { 16 ESPaDOnS observations (this work) }\end{array}$ & 12 ESPaDOnS observations (this work) \\
Photometry & SAO RAS time series (this work) & Amateur astronomer time series (Gary et al. 2013) \\
\hline
\end{tabular}

Notes. LR, MR, HR stand for low-, mid-, high-resolution, respectively.

observing programmes and are presented here for the first time, and some have been taken from ESO archive and/or have already been published (either by ourselves or by other groups).

Our general observing plan was first to identify a candidate weak field magnetic star (either from the literature or using data from our surveys), then to try to detect variability either in intensity or in polarisation spectra with a small number of observations per star, and finally, when variations were found, to monitor the star with a cadence suitable to detect a period in the range of hours to weeks. This required a series of observations spaced one or a few days apart, combined with either some repeated observations per night (WD 2047+372) or at least observations at a range of hour angles (WD 2359-434). ESPaDOnS at the CHFT (see Sect. 3.3 below) was the principal instrument used for this longer-term monitoring. Photometric data were also used to confirm/identify a rotation period.

\subsection{Mid-resolution spectropolarimetry with MSS of the SAO $6 m$ telescope}

The MSS is a moderate-beam classic spectrograph equipped with a circular polarisation analyser combined with a seven-layer image slicer (Chountonov 2004) permanently installed at the $6 \mathrm{~m}$ telescope (BTA) at the Special Astrophysical Observatory (SAO) of the Russian Academy of Sciences (RAS). The description of the optical scheme of the MSS and its capabilities can be found in the web page of the instrument ${ }^{1}$. The rather dated design of the instrument, which has several mirrors along the beam path, seriously reduces total efficiency in high-resolution observations of comparatively faint white dwarfs. To respond to this problem the observatory staff is currently constructing a modern, highresolution spectropolarimeter which is expected to be commissioned within a couple of years; see Valyavin et al. (2015). With this instrument it will be possible to extend BTA observations of white dwarfs up to $m_{V} \sim 15$ or fainter. Our current observational limit with the high-resolution spectropolarimeters of the $6 \mathrm{~m}$ telescope is around $V \sim 13$. Nevertheless we decided to use this instrument in order to obtain as much information about WD 2047+372 as possible, for which we obtained four spectropolarimetric observations in the course of two consecutive nights, 2016-06-12 and 13. The MSS spectropolarimeter was used with a spectral resolving power of $R=10000$ at $\mathrm{H} \alpha$. The observations were conducted following the standard procedures that we use in observations with BTA (see e.g. Valyavin et al. 2005). A polarisation observation consists of a series of paired exposures obtained at two orthogonal orientations of the quarter

\footnotetext{
1 http://www.sao.ru/hq/lizm/mss/en/
}

wave-plate. Data reduction and field measurements are also standard and summarised by Landstreet et al. (2015). The log of MSS observations is given in Table 4.

\subsection{Low-resolution spectropolarimetry with FORS of the ESO VLT}

FORS1 and FORS2 (Appenzeller et al. 1998) are twin multipurpose instruments capable of doing imaging and low-resolution spectroscopy attached at the cassegrain focus of the $8 \mathrm{~m}$ units of the ESO VLT. When both instruments were operating, FORS1 was equipped with polarimetric optics. Polarimetric optics were then moved to FORS2 when FORS1 was decommissioned in 2008. Both instruments have been used for observations of WDs by several authors. In this paper we consider UVES and FORS1 archive data of WD 2359-434 as well as four new observations obtained during our survey. Our new spectra cover the wavelength window between about 3670 and $5120 \AA$ and have an effective resolving power of about 2200 . They have a typical $\mathrm{S} / \mathrm{N}$ ratio per pixel of between 270 and 400 per $\AA$. These data were reduced, and the mean longitudinal field $\left\langle B_{z}\right\rangle$ deduced, as described in detail by Bagnulo et al. (2015): for these spectra $\left\langle B_{z}\right\rangle$ was determined by measuring the slope of the correlation between the circular polarisation $V(\lambda)$ and $(1 / I)(\mathrm{d} I(\lambda) / \mathrm{d} \lambda)$ (see Sect. 4.3). The log of FORS2 observations is given in Table 5.

\subsection{High-resolution spectropolarimetry with ESPaDOnS of the CHFT}

In order to study the magnetic fields of the two super-weak field MWDs investigated in this paper in more detail, we obtained observations with the high-resolution CFHT spectropolarimeter ESPaDOnS, which we had previously found to be a very powerful tool for studying fields in bright DA WDs (Landstreet et al. 2015, 2016). The instrument provides highprecision, high-resolution spectropolarimetry over nearly the complete spectrum from $3800 \AA$ to $1.04 \mu \mathrm{m}$ using a polarimetric module above the entrance apertures of a cross-dispersed echelle spectrograph, as briefly described by the instrument web page $^{2}$ and by Landstreet et al. (2008). ESPaDOnS has very high throughput, but with $R=65000$ on a $3.6 \mathrm{~m}$ telescope, observing even relatively bright WDs is uncomfortably near the magnitude limit set by cosmic rays, CCD read noise, sky brightness near twilight or when the moon is near full, and one needs to keep each observation to a minimum duration in order to

2 http://www.cfht.hawaii.edu/Instruments/Spectroscopy/ Espadons 
Table 3. Journal of photometric observations of WD $2047+372$ with the $1 \mathrm{~m}$ telescope of the SAO-RAS.

\begin{tabular}{llr}
\hline \hline Date & JD (days) & $N_{\exp }$ \\
\hline $2016-08-19$ & $2457620.31-20.57$ & 1083 \\
$2016-09-12$ & $2457644.33-44.39$ & 238 \\
$2016-09-19$ & $2457651.22-51.41$ & 513 \\
\hline
\end{tabular}

Table 4. Journal of observations of WD $2047+372$.

\begin{tabular}{llrrr}
\hline \hline Instrument & MJD & $\begin{array}{r}\text { Exp. } \\
(\mathrm{s})\end{array}$ & \multicolumn{1}{c}{$\begin{array}{c}\left\langle B_{z}\right\rangle \\
(\mathrm{kG})\end{array}$} & $\begin{array}{c}\langle|B|\rangle \\
(\mathrm{kG})\end{array}$ \\
\hline ISIS & 57266.082 & 3360 & $0.61 \pm 0.24$ & \\
E 1844823 & 57326.255 & 3256 & $6.00 \pm 1.67$ & $57.4 \pm 2.5$ \\
E 1939267 & 57549.554 & 4416 & $-10.79 \pm 1.37$ & $59.8 \pm 2.5$ \\
E 1939614 & 57551.601 & 4416 & $16.11 \pm 2.77$ & $61.3 \pm 3.0$ \\
MSS & 57551.942 & 3600 & $2.20 \pm 2.00$ & \\
MSS & 57551.984 & 3660 & $-2.80 \pm 2.00$ & \\
MSS & 57552.901 & 3660 & $-9.70 \pm 1.80$ & \\
MSS & 57552.944 & 3660 & $-7.60 \pm 2.30$ & \\
E 1940930 & 57558.609 & 4416 & $-1.82 \pm 1.13$ & $55.3 \pm 2.5$ \\
E 1941326 & 57560.511 & 4416 & $-11.69 \pm 1.72$ & $59.5 \pm 2.5$ \\
E 1941535 & 57561.500 & 4416 & $-9.30 \pm 1.26$ & $60.5 \pm 2.5$ \\
E 1941724 & 57562.451 & 3400 & $-9.57 \pm 1.60$ & $59.5 \pm 2.5$ \\
E 1941744 & 57562.611 & 3400 & $8.97 \pm 1.59$ & $57.4 \pm 2.5$ \\
E 1974328 & 57603.483 & 3360 & $3.25 \pm 1.31$ & $58.9 \pm 2.5$ \\
E 1974340 & 57603.577 & 3360 & $-6.46 \pm 1.55$ & $63.0 \pm 2.5$ \\
E 1974958 & 57606.380 & 3360 & $6.89 \pm 1.46$ & $60.4 \pm 2.5$ \\
E 1974982 & 57606.553 & 3360 & $13.44 \pm 2.03$ & $61.9 \pm 2.5$ \\
E 1975651 & 57609.499 & 3360 & $14.66 \pm 1.51$ & $60.2 \pm 2.5$ \\
E 1976104 & 57611.406 & 3360 & $6.16 \pm 1.23$ & $57.9 \pm 2.5$ \\
E 1976387 & 57612.556 & 3360 & $-10.97 \pm 1.38$ & $61.5 \pm 2.5$ \\
E 1976638 & 57613.487 & 3360 & $-8.65 \pm 1.23$ & $64.4 \pm 2.5$ \\
E 1977043 & 57615.441 & 3360 & $-10.32 \pm 1.34$ & $58.8 \pm 2.5$ \\
\hline
\end{tabular}

Notes. Column 1 gives the instrument used (ESPaDOnS spectra are identified by the letter $\mathrm{E}$ followed by the identification number); Col. 2 gives the Modified Julian Date at mid-exposure, Col. 3 the exposure time, Col. 4 the measured longitudinal field $\left\langle B_{z}\right\rangle$ with its uncertainty, and Col. 5 the mean field modulus $\langle|B|\rangle$ and its estimated uncertainty.

sample a reasonable range of probable rotation periods. As a compromise, each observation (comprising four sub-exposures taken at different wave-plate positions) lasted about an hour in total. This integration time was found (after binning spectra to $R \approx 15000$ ) to provide sufficient $\mathrm{S} / \mathrm{N}$ to clearly see magnetic effects in the deep and sharp non-LTE cores of the $\mathrm{H} \alpha$ lines of the two MWDs with field uncertainties of the order of one or a few kG (Landstreet et al. 2015, 2016).

The CFHT allocated time during the 2016A and 2016B semesters, and with the flexibility and excellent consistency provided by the queue service observing team, we were able to obtain two series of polarised spectra. We note that the series of ESPaDOnS spectra obtained for WD 2359-434 is particularly remarkable, because the star's position at $-43^{\circ}$ guaranteed that all spectra were taken at more than two air masses. In spite of this, nine spectra were obtained during ten nights, over a sufficient range in hour angle to make possible the secure discovery of periodic magnetic variations with a period of about $2.6 \mathrm{~h}$ (see below). The log of ESPaDOnS observations is given in Tables 4 and 5.

\subsection{Photometry with the SAO $1 \mathrm{~m}$ telescope}

In order to supplement our magnetic measurements, we have also conducted photometric observations of WD 2047+372.
Table 5. Journal of observations of WD 2359-434.

\begin{tabular}{llrcrr}
\hline \hline Instrument & MJD & $\begin{array}{r}\text { Exp. } \\
(\mathrm{s})\end{array}$ & $\begin{array}{c}\left\langle B_{z}\right\rangle \\
(\mathrm{kG})\end{array}$ & $\begin{array}{r}\langle|B|\rangle \\
(\mathrm{kG})\end{array}$ & $\begin{array}{r}W_{\lambda} \\
(\mathrm{mA})\end{array}$ \\
\hline UVES & 51739.276 & 300 & & 110 & \\
UVES & 51743.301 & 300 & & 110 & \\
FORS1 & 52583.025 & 2188 & $4.10 \pm 0.84$ & & \\
FORS1 & 52608.056 & 2188 & $3.09 \pm 0.51$ & & \\
FORS2 & 57234.299 & 4296 & $2.14 \pm 0.37$ & & \\
FORS2 & 57273.252 & 4296 & $2.94 \pm 0.42$ & & \\
E 1844491 & 57324.324 & 3256 & $10.81 \pm 3.28$ & 99 & 38.4 \\
E 1844835 & 57326.343 & 3256 & $3.50 \pm 2.86$ & 99 & 17.5 \\
E 1844989 & 57327.289 & 3256 & $4.80 \pm 1.76$ & 57 & 304.7 \\
E 1845170 & 57328.285 & 3256 & $5.70 \pm 2.15$ & 74 & 212.8 \\
FORS2 & 57544.374 & 2496 & $2.40 \pm 0.40$ & & \\
FORS2 & 57567.399 & 2496 & $2.10 \pm 0.34$ & & \\
E 1974336 & 57603.533 & 3360 & $6.69 \pm 1.80$ & 47 & 347.7 \\
E 1974574 & 57604.542 & 3360 & $-0.59 \pm 2.03$ & 49 & 317.6 \\
E 1974986 & 57606.598 & 3360 & $5.43 \pm 2.59$ & 76 & 152.9 \\
E 1975449 & 57608.493 & 3360 & $8.37 \pm 2.06$ & 61 & 234.6 \\
E 1975659 & 57609.546 & 3360 & $8.57 \pm 3.46$ & 100 & -12.3 \\
E 1975887 & 57610.585 & 3360 & $8.89 \pm 2.41$ & 71 & 164.8 \\
E 1976112 & 57611.476 & 3360 & $5.69 \pm 2.07$ & 73 & 150.9 \\
E 1976391 & 57612.599 & 3360 & $7.49 \pm 2.51$ & 81 & 113.2 \\
E 1976646 & 57613.556 & 3360 & $6.23 \pm 2.50$ & 67 & 212.2 \\
\hline
\end{tabular}

Notes. Column 1 gives the instrument used (ESPaDOnS data are identified by the letter $\mathrm{E}$ followed by the identification number), Col. 2 the Modified Julian Date at mid-exposure, Col. 3 the exposure time, Col. 4 the measured longitudinal field $\left\langle B_{z}\right\rangle$ with its uncertainty, and Col. 5 the mean field modulus in $\mathrm{kG}$ (which has a typical estimated uncertainty of $10 \mathrm{kG})$, and Col. 6 the measured $\mathrm{H} \alpha$ line core $\mathrm{EW} W_{\lambda} \cdot\langle|B|\rangle$ values for UVES spectra are from Koester et al. (2009).

These observations were obtained during three nights (August 19, September 12 and September 19, 2016) with the $1 \mathrm{~m}$ telescope of the SAO of the RAS (see Table 3). All observations were conducted in the broadband $m_{V}$ filter of the Johnson system as a continuous run of short duration individual exposures, typically $20-30 \mathrm{~s}$ in length. Before each night of observations, exposures of scattered sunlight were made during twilight for flatfield correction. Photometric data reduction was carried out with software written in the Python programming language using the standard package for astrophysical data reduction from the Image Reduction and Analysis Facility (IRAF) of the U.S. National Optical Astronomy Observatories.

\subsection{Amateur astronomer photometry}

Broad-band photometric monitoring of WD 2359-434 has been undertaken by a group of amateur astronomers, particularly by T.G. Tan in Perth, Australia, using telescopes of around $30 \mathrm{~cm}$ aperture. This work was initiated as an effort to detect planetary transits in white dwarf systems. When sinusoidal light variations of WD 2359-434 were found, these were followed up. A major observing campaign was carried out on this star during 2011 and 2012, involving many tens of hours of observation (Gary et al. 2013). Further data were acquired during 2013, 2014, and $2016^{3}$. Most of the observations were carried out in either the $R c$ or $B$ photometric bands. The precision of individual photometry measurements on good nights, with integration times of order $1 \mathrm{~min}$, is in the vicinity of 3-5 mmag.

3 http://brucegary.net/WDE/WD2359-434/WD2359-434.htm 

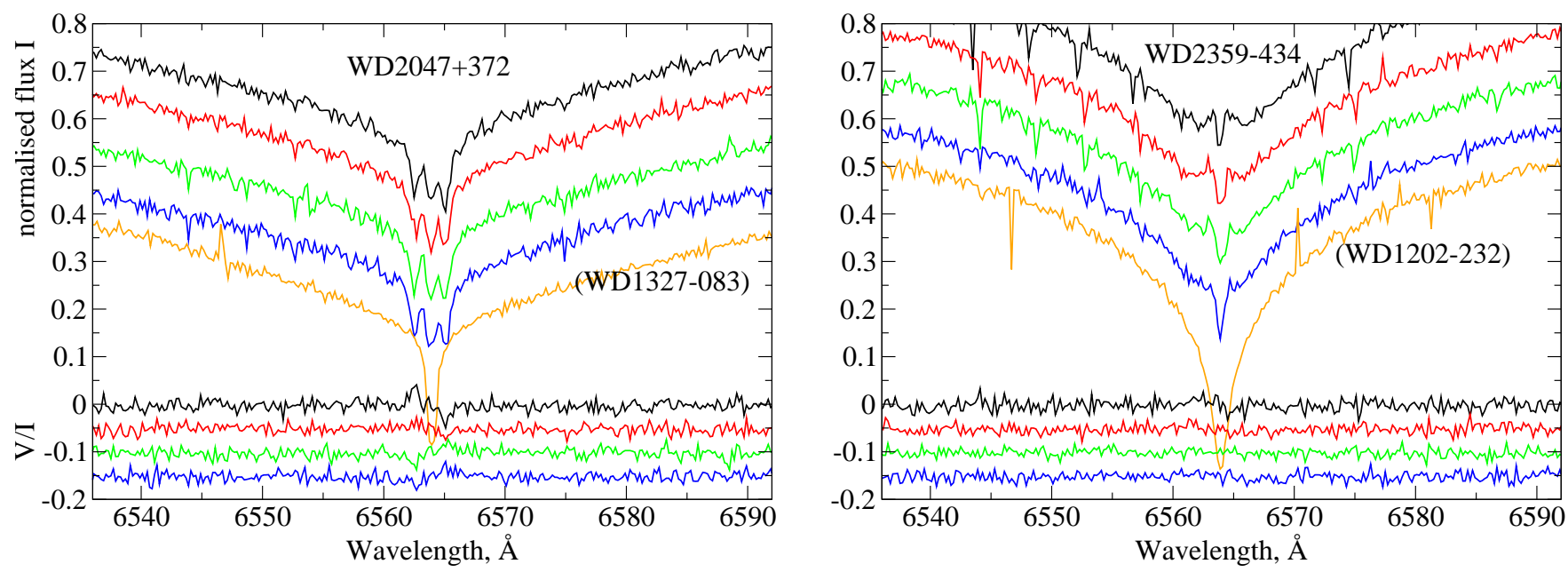

Fig. 1. ESPaDOnS spectra of WD 2047+372 (left panel) and WD 2359-434 (right panel). Both panels are organised in a similar way. The top four solid lines show the intensity $I$ spectra, and the lowest solid lines represent the corresponding circular polarisation $V$ spectra of the H $\alpha$ region. For WD 2047+372 the spectra numbers are 1975651, 1976104, 1976387, 1976638; for WD 2359-434 the spectra are 1975659, 1976391, 1976646, and 1974336. In between the four ESPaDOnS $I$ spectra is a UVES $I$ spectrum of a non-magnetic star with similar effective temperature as the magnetic star: the comparison star for WD 2047+372 is WD 1327-083, and the comparison star for WD 2359-434 is WD 1202-232. Data are binned in $0.2 \AA$ boxes to improve $\mathrm{S} / \mathrm{N}$. All $I$ spectra are normalised to 1.0 at $\pm 200 \AA$ from $\mathrm{H} \alpha$ line centre, then shifted down from top $I$ spectrum in steps of 0.1 for visibility. All $V$ spectra are normalised to 0.0 outside the line core, then shifted down from top $V$ spectrum in steps of 0.05 .

\section{Measurements of spectropolarimetric data}

Our spectral database includes high-resolution ESPaDOnS polarised spectra for both stars. These spectra can be conveniently used for a preliminary quantitative comparison of the magnetic fields of the two stars.

Most of the useful information in the ESPaDOnS spectra about the fields of the two MWDs is found within a few $\AA$ of the centre of $\mathrm{H} \alpha$. Four representative flux spectra (Stokes $I$ spectra) of WD 2047+372 in this spectral region are shown in the left panel of Fig. 1, together with a comparison spectrum of the nonmagnetic and unpolarised star WD 1327-083 ( $\left.T_{\text {eff }}=14570 \mathrm{~K}\right)$, and four typical spectra of WD 2359-434 are shown in the right panel of Fig. 1, together with a UVES comparison spectrum of the non-magnetic star WD 1202-232 ( $\left.T_{\text {eff }}=8770 \mathrm{~K}\right)$.

In both stars, clear (but quite different) Zeeman splitting is visible in the intensity spectra. In WD 2047+372 the Zeeman pattern contains sharp $\sigma$ components, very similar in shape to the central $\pi$ components. The shape of the clear and simple Zeeman flux triplet pattern hardly varies from one observation to another. Significantly non-zero Zeeman polarisation signals, varying from one spectrum to another, are visible to the eye in most of the Stokes $V$ (circular polarisation) spectra of WD 2047+372. In contrast, only the central $\pi$ component of the Zeeman split line core is obvious in WD 2359-434, while the two $\sigma$ components are broad and very poorly defined. The entire shape of the line core of this star is variable. In the ESPaDOnS $V$ spectra of WD 2359-434, non-zero Zeeman polarisation signals are barely detectable to the eye, although non-zero values of $\left\langle B_{z}\right\rangle$ are securely detected at the several sigma level in all six FORS polarised spectra.

The ESPaDOnS spectra were used to extract the following information:

Variability of the $\mathrm{H} \alpha$ line core profile.

The equivalent width of $\mathrm{H} \alpha$.

The mean longitudinal magnetic field $\left\langle B_{z}\right\rangle$.

The mean magnetic field modulus $\langle|B|\rangle$.
FORS low-resolution-, and ISIS and MSS mid-resolution spectra, were used only to measure the mean longitudinal magnetic field.

In the following we describe these measurements, and we report the results obtained for the two stars. Our measurements will be used below (Sects. 5 and 6) to confirm stellar variability, search for its period, and finally to obtain approximate magnetic models of our target stars.

\subsection{Line core variability}

The high-resolution ESPaDOnS spectra may be used to test whether the $\mathrm{H} \alpha$ line core is detectably variable or not as follows. We first construct a mean inner $\mathrm{H} \alpha$ line by averaging all available spectra. This averaging is carried out on the normalised spectra provided by the CFHT's Libre-Esprit reduction pipeline, which produces spectra that are consistently normalised in a very similar way. Individual spectra are then slightly rescaled to coincide as well as possible with the mean spectrum. After rescaling, the standard deviation $\sigma_{\text {csp }}\left(\lambda_{j}\right)$ of the ensemble of spectra is calculated point by point as a function of $\lambda_{j}$ through the profile using

$\sigma_{\mathrm{sp}}\left(\lambda_{j}\right)=\left\{\frac{\sum_{i=1}^{n}\left[y_{i}\left(\lambda_{j}\right)-y_{\mathrm{m}}\left(\lambda_{j}\right)\right]^{2}}{n-1}\right\}^{1 / 2}$,

where $i$ is summed over the $n=16$ spectra at each point in the spectrum, and $y_{\mathrm{m}}\left(\lambda_{j}\right)$ is the value of the mean spectrum at that wavelength.

This mean spectrum of WD $2047+372$ is shown in the left panel of Fig. 2. The variation of $\sigma_{\mathrm{sp}}\left(\lambda_{j}\right)$ across the core of $\mathrm{H} \alpha$ is shown below the mean $I$ line profile (shifted upward by +0.4 from its original value close to zero, for ease of comparison with the $I$ profile; the zero line has also been shifted upward by +0.4 and is seen about 0.015 below the computed standard deviation). The striking feature of the variation of $\sigma_{\mathrm{sp}}$ across the line core where the Zeeman triplet is found is that there is no evidence for larger dispersion. This confirms what is anticipated above, that 

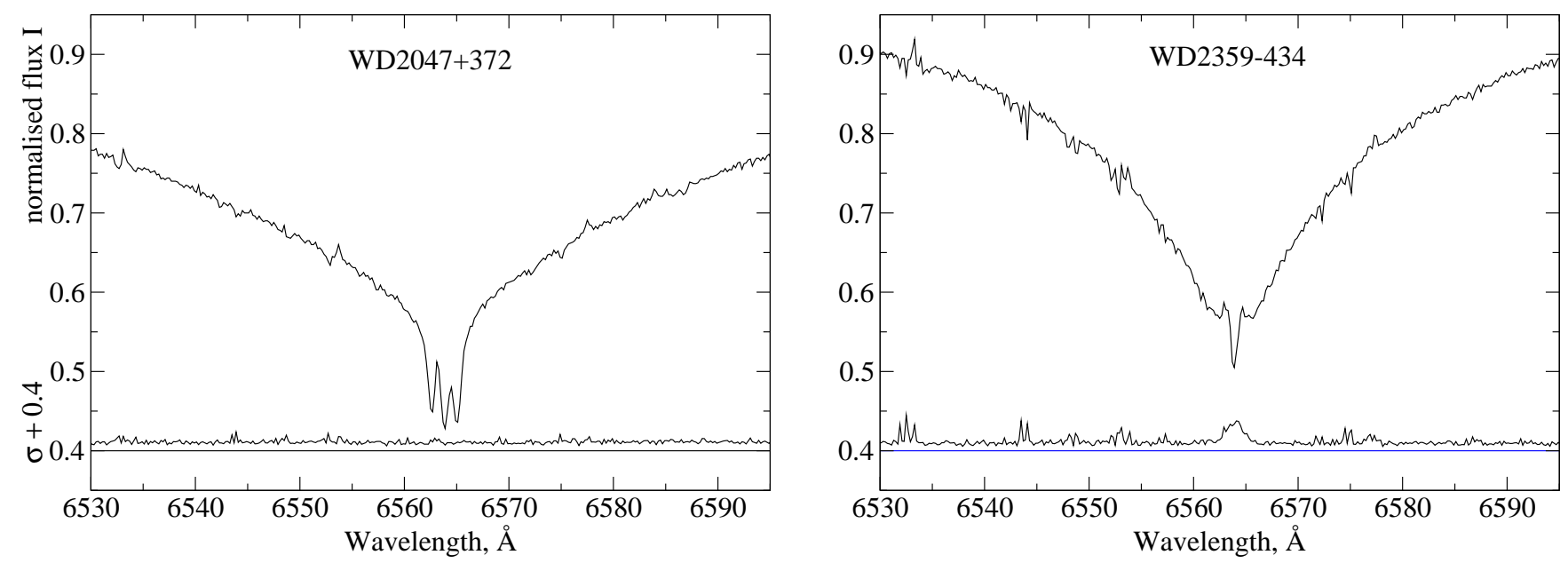

Fig. 2. Mean $\mathrm{H} \alpha$ flux $(I)$ spectrum computed from all individual spectra, with standard deviation $\sigma$ of individual spectra around the mean, shifted by +0.4 for clarity. The straight line at +0.4 is the zero point for $\sigma$, also shifted upward by +0.4 . The left panel refers to WD $2047+372$ (excluding spectrum E 1939614) and the right panel refers to WD 2359-434.

is, that the available $\mathrm{H} \alpha$ spectra show no evidence that the value of $\langle|B|\rangle$ varies significantly as the WD rotates; $\langle|B|\rangle$ is constant at about $60 \mathrm{kG}$.

In contrast to WD 2047+372, WD 2359-434 (right panel of Fig. 2) exhibits a clear increase in dispersion across the core of $\mathrm{H} \alpha$ (and also across several of the weak atmospheric water vapour absorption lines present in the stellar line wings). This variability is also visible in the $I$ spectra of Fig. 1 .

\subsection{Equivalent width}

The simplest moment of the $\mathrm{H} \alpha$ I profile is the equivalent width of the Zeeman-split core. In the context of this work, EW measurements serve to detect periodic variability. They were found useful only in the case of WD 2359-434, for which they are also found to act as proxies for $\langle|B|\rangle$. To obtain $\mathrm{EW}$ measurements, we first renormalised each $\mathrm{ESPaDOnS} \mathrm{H} \alpha$ profile (as produced in normalised form by the CFHT reduction programme LibreEsprit) to minimise the mean squared difference from the mean of all $I \mathrm{H} \alpha$ spectra, using the two wing intervals $6530-6560 \AA$ and 6568-6598 $\AA$ (omitting small intervals with significantly variable atmospheric absorption). This led only to small amounts of rescaling, with changes of $2 \%$ or less. We then integrated the core area between $6560 \AA$ and $6567.8 \AA$ relative to an arbitrary "continuum" level at 0.6 . The resulting equivalent width measures $W_{\lambda}$, in $\mathrm{m} \AA$, are reported in Table 5. We note that although the values have an arbitrary zero point (unlike the situation for a sharp line isolated in a local continuum), the "variations" have the same meaning as usual for equivalent width variations. We estimate that the total uncertainty of each of these measurements is roughly $20 \mathrm{~m} \AA$, mainly due to uncertainty in the slight renormalisation of the spectra to a common mean.

\subsection{The mean longitudinal magnetic field}

The mean longitudinal magnetic field can be estimated in two different ways. From mid- and low-resolution spectra we have used the relationship between $V(\lambda / I)$ and $(1 / I)(\mathrm{d} I(\lambda) / \mathrm{d} \lambda)($ e.g. Bagnulo et al. 2015). From high-resolution ESPaDOnS spectra of $\mathrm{H} \alpha$, the mean longitudinal field can conveniently be derived by measuring the separation between the wavelength of the centroid of the line core profile as seen in right polarised light and the wavelength of the centroid of the line core in left circularly polarised light (Mathys 1989; Donati et al. 1997). $\left\langle B_{z}\right\rangle$ was computed separately for the $\mathrm{H} \alpha$ and $\mathrm{H} \beta$ lines. For WD $2047+372$ the integration limits were 6561.5 and $6566.0 \AA$ for $\mathrm{H} \alpha$, and 4860.0 and $4864.2 \AA$ for $\mathrm{H} \beta$. The two $\left\langle B_{z}\right\rangle$ values were then combined by weighted averaging. We note that, in general, the value of $\left\langle B_{z}\right\rangle$ from $\mathrm{H} \beta$ has an uncertainty approximately three times larger than the value derived from $\mathrm{H} \alpha$, so the contribution of $\mathrm{H} \beta$ to the final values is almost insignificant. Details of the numerical application of this method to WD ESPaDOnS spectra are discussed by Landstreet et al. (2015).

The values of $\left\langle B_{z}\right\rangle$ derived from each of the spectra of WD 2047+372 are reported in the third column of Table 4 (we note that the ISIS measurement and the first ESPaDOnS measurement were already published by Landstreet et al. 2016). In 15 of 17 measurements obtained with ESPaDOnS, the field is detected at more than the $3 \sigma_{B_{z}}$ level. The values of the uncertainty $\sigma_{B_{z}}$ are generally between 1 and $2 \mathrm{kG}$, so the value of $\left\langle B_{z}\right\rangle$ varies by several times $\sigma_{B_{z}}$ on each side of zero. Thus these data are well-adapted to searching for time variability. We note that the ISIS measurement has far higher precision than the ESPaDOnS measurements.

For WD 2359-434, the integration limits to use in determination of $\left\langle B_{z}\right\rangle$ are not obvious; the sigma components blend very smoothly into the broader line wings. For this star, the integration limits were chosen to be just a little beyond the edges of the apparent region of spectral variation (and the limit of non-zero circular polarisation in an averaged spectrum computed for several spectra obtained around maximum field). The limits chosen were 4859.5 and $4864.7 \AA$ for $\mathrm{H} \beta$, and 6559.0 and $6568.6 \AA$ for $\mathrm{H} \alpha$.

The values of $\left\langle B_{z}\right\rangle$ derived from each of the spectra of WD 2359-434 are reported in Col. 3 of Table 5. It is clear that the FORS measurements have much smaller error bars than those obtained with ESPaDOnS and MSS. The large uncertainties of the ESPaDOnS measurements relative to the FORS measurements are a consequence of the fact that for this star we are measuring the small wavelength shift of a single broad, shallow feature between the right and left circularly polarised spectra, using only a few $\AA$ around the centre of $\mathrm{H} \alpha$; in contrast, in the FORS data we are also measuring about the same small shift 
using broad line wings, but in the FORS data the full wings of five Balmer lines (starting with $\mathrm{H} \beta$ ) contribute to the $\mathrm{S} / \mathrm{N}$ of the measurement.

\subsection{The mean magnetic field modulus}

Another measurement of direct interest for characterising the surface magnetic field is the mean field modulus $\langle|B|\rangle$, a quantity representing the local surface values of $|B|$, averaged over the visible hemisphere of the star. This field moment is determined by measuring the separation of the two outer $(\sigma)$ Zeeman components of the magnetically split line from the central $(\pi)$ component, and deriving the value of $\langle|B|\rangle$ by using the expression (adapted from the equations describing the Zeeman effect)

$\Delta \lambda_{\mathrm{Z}}=4.67 \times 10^{-13} \bar{g} \lambda_{0}^{2}\langle|B|\rangle$,

where $\Delta \lambda_{\mathrm{Z}}$ is the $\pi-\sigma$ separation, $\lambda_{0}$ is the wavelength of the spectral line in the absence of a magnetic field, $\langle|B|\rangle$ is the magnetic field strength, and $\bar{g}$ is the mean Landé factor (the mean shift of the Zeeman $\sigma$ components from the unperturbed line wavelength, measured in units of the classical Zeeman splitting). For $\mathrm{H} \alpha, \bar{g}=1$ (Casini \& Landi Degl'Innocenti 1994). In Eq. (2) all wavelengths are in $\AA$ units, and field strength is measured in Gauss (G). We note that we usually actually measure the separation of the two $\sigma$ components, which is twice the separation described by Eq. (2).

In the discovery spectrum of the field of WD 2047+372 we estimated the value of $\langle|B|\rangle=56.9 \pm 0.4 \mathrm{kG}$, where the uncertainty was simply an estimate of the uncertainty of repeatedly measuring the $\sigma-\pi$ separation wavelengths, using Gaussian fits to the three Zeeman components. We have measured the value of $\langle|B|\rangle$ for all the ESPaDOnS spectra using the separation between the two $\sigma$ components, where the centroid wavelength of each $\sigma$ component was measured using the $e$ - $e$ command of the IRAF spectrum plotting and measuring function splot. The resulting $\langle|B|\rangle$ measurements are reported in Table 4 . It is clear on inspection that the values of $\langle|B|\rangle$ scatter around a mean of about $60 \mathrm{kG}$ with approximately the scatter expected from our estimates of measurement uncertainty (based on experiments with different ways of measuring the $\sigma-\sigma$ separation). We note that the measurement uncertainties reported for $\langle|B|\rangle$ now effectively include an uncertainty for the transformation from $\sigma-\sigma$ component separation to $\langle|B|\rangle$, and hence are significantly larger than the uncertainty reported in our initial paper on WD 2047+372. There is no convincing evidence in these $\langle|B|\rangle$ data that $\langle|B|\rangle$ in this MWD varies significantly.

The Zeeman patterns of WD 2359-434 are remarkably different from those of WD 2047+372, although both stars appear to have fields of somewhat less than $100 \mathrm{kG}$. Where the Zeeman $\sigma$ components of WD 2047+372 are not significantly wider than the $\pi$ component, those of WD 2359-434 are so broad that identifying them is difficult. It seems clear that the local variations in the value of $|B|$ seen on at least part of the stellar surface reach a factor of two or even more, while on WD 2047+372 it is not obvious that $|B|$ varies at all over a visible hemisphere.

As a result, in WD 2359-434 it is very difficult to know how to measure the field moment $\langle|B|\rangle$, as in this star the $\sigma$ components of the Zeeman triplet are broad and shallow, have unfamiliar shapes, have low $\mathrm{S} / \mathrm{N}$ relative to the overall profile, and blend imperceptibly into the line wings. However, close examination of the individual spectra strongly suggests that the positions of the Zeeman $\sigma$ components varies considerably from one spectrum to another, from positions well clear of the central $\pi$ component to almost merging with the $\pi$ component. Thus it appears that in this $\operatorname{star}\langle|B|\rangle$ is rather strongly variable.

We experimented with a number of methods of obtaining reproducible and meaningful measurements of $\langle|B|\rangle$. Reasonable estimates by eye of the positions of the $\sigma$ components of the line profiles could be made for some of the spectra, especially those in which the $\sigma$ components are relatively obvious (e.g. the top spectrum in Fig. 1). However, this method was often foiled by noise (even in spectra smoothed to $0.2 \AA$ bins) because of the very shallow shape of these components. The resulting measurements were found to be too noisy to be very useful, although they do suggest that the value of $\langle|B|\rangle$ varies between nearly $100 \mathrm{kG}$ and below approximately $60 \mathrm{kG}$.

Using $e-e$ command of the IRAF splot function allowed us to estimate the position of the centroid of each of the $\sigma$ components of the $\mathrm{H} \alpha$ line core in the various spectra, but this quantity showed only very small variations in the deduced values of $\langle|B|\rangle$ (roughly from 100 to $80 \mathrm{kG}$ ) in spite of the obvious variations of the line shapes. We concluded that this particular measure is not very sensitive to the value of $\langle|B|\rangle$.

Table 5 shows that one measured quantity, our measure of the equivalent width $W_{\lambda}$ of the line core in Stokes $I$, varies from spectrum to spectrum by much more than the associated uncertainty. We therefore considered using this quantity as a proxy for $\langle|B|\rangle$. Inspection of individual spectra clearly shows that $W_{\lambda}$ is closely associated with the form of the $\sigma$ components. In spectra in which the $\sigma$ components are relatively clear and well separated from the $\pi$ component, the line core is broad and shallow, and $W_{\lambda}$ is small; when the $\sigma$ components are close to the central $\pi$ component, the line core is deeper and $W_{\lambda}$ is relatively large. We then experimented with averages of spectra of similar $W_{\lambda}$ values and apparently similar form to obtain averaged spectra of small, intermediate, and large values of $W_{\lambda}$ of higher $\mathrm{S} / \mathrm{N}$ than the individual spectra. With these averaged spectra of improved $\mathrm{S} / \mathrm{N}$ we were able to estimate more reliably the $\sigma-\sigma$ separation, and to then estimate $\langle|B|\rangle$. We then associated the deduced value of $\langle|B|\rangle$ for each averaged spectrum with the average value of $W_{\lambda}$.

In this way we found that an average of the three spectra with $W_{\lambda} \approx 20 \mathrm{~m} \AA$ appear to have $\langle|B|\rangle \approx 95 \mathrm{kG}$; an average of three spectra with $W_{\lambda} \approx 175 \mathrm{~m} \AA$ have $\langle|B|\rangle \approx 72.5 \mathrm{kG}$; and we estimate that the two averaged spectra with $W_{\lambda} \approx 330 \mathrm{~m} \AA$ correspond to $\langle|B|\rangle \sim 50 \mathrm{kG}$, although this value is more uncertain than the other two $\langle|B|\rangle$ values. If we make the further assumption that $W_{\lambda}$ is (very roughly) linearly related to $\langle|B|\rangle$, we can use the simple relation,

$\langle|B|\rangle \approx 50+45 \frac{330-W_{\lambda}}{310} \mathrm{kG}$,

to estimate the value of $\langle|B|\rangle$ to associate with each spectrum. Estimated values of $\langle|B|\rangle$ computed in this manner are reported in Col. 5 of Table 5. We consider that realistically each of these values is uncertain by roughly $10 \mathrm{kG}$.

\section{Search for stellar rotation period}

It is clear from the observational material that both WD 2047+372 and WD 2359-434 are variable. Judging from a large number of previously studied MWDs, the variations in the Zeeman line profiles that we observe are due to the rotation of these stars, and are therefore periodic.

The next step is to search the moment data for each star for periodic variations. This has been done using the FORTRAN 
program DCHI.F, already employed for many years for period searches on magnetic field and photometric data for main sequence magnetic stars (e.g. Neiner et al. 2012; Landstreet et al. 2014). The program starts with a list of $n$ measurements, for example $\left\langle B_{z}\right\rangle_{i}$, with the associated uncertainty $\sigma_{i}$ and Modified Julian Date $\mathrm{MJD}_{i}$. For a given frequency (or period), the program computes phases of all the data points, and then carries out a least-squares fit to the observational data using a sine wave whose zero point, amplitude, and phase are varied. The quality of the fit is assessed by minimising the value the reduced chisquared parameter,

$\chi^{2} / v=\frac{\sum_{i=1}^{n}\left[\left(y_{i}-y_{\mathrm{fit}}\right) / \sigma_{i}\right]^{2}}{n-3}$,

at that frequency. This quantity is then computed for all independent frequencies between two given limits (with a sampling spacing chosen by the user), and the resulting value of $\chi^{2} / v$ of the best fit found at each period is output as a function of the period. If the data vary periodically in an approximately sinusoidal manner, and the measurement uncertainties are realistic, the correct period will have a best fit $\chi^{2} / v$ value of about 1 . If the sampling of the data is sufficiently dense and well spaced, the correct value of the period may be identified uniquely, but often there are aliasing problems, especially due to observations spaced at a larger interval of time than the period.

\subsection{The rotation period of WD $2047+372$}

To estimate the period of WD 2047+372 we searched between 10 and $0.1 \mathrm{~d}$ for periodic sinusoidal variation of the $\left\langle B_{z}\right\rangle$ measurements of WD 2047+372 from 2016 (MJDs starting with 57 549), which are closely spaced. In the range of frequencies searched we found that there is only a single frequency that gives a really good sine-wave fit to the data, at a frequency $f=$ $4.1124 \pm 0.0012$ cycles $/ \mathrm{d}$, or a period of $P=0.24317 \pm 0.00007 \mathrm{~d}$. Two frequency aliases at approximately 3 and 5 cycles/d, caused by changing the cycle count between successive nights by \pm 1 , are strongly rejected because we have multiple observations of $\left\langle B_{z}\right\rangle$ on three separate nights.

When the first ESPaDOnS observation, taken about seven months before the remaining ones, is added to the data set, the frequency discrimination is substantially sharper and selects uniquely the frequency $f=4.11238 \pm 0.00044$, or equivalently the period $P=0.243168 \pm 0.000024$, values that are about three times as precise as the values derived from the shorter data sequence. A portion of the $\chi^{2} / v$ spectrum of the full data set is shown in the top panel of Fig. 3. Using the best-fit identified by our program, we find that the rotational ephemeris of WD $2047+372$ is given by

$\operatorname{MJD}\left(\max \left\langle B_{z}\right\rangle\right)=57326.209+(0.243168 \pm 0.000024) \times E$,

where phase $=0^{\circ}$ corresponds to the maximum of the mean longitudinal magnetic field.

The $\left\langle B_{z}\right\rangle$ values for WD $2047+372$ can now be plotted using the best-fit period. The result is shown in the middle panel of Fig. 3. It is clear that the variation of $\left\langle B_{z}\right\rangle$ with phase, using the best period, is indeed quite sinusoidal. In fact, it seems obvious from the figure, in which only two data points appear to miss the fitted curve by substantially more than one standard error, that the uncertainties computed for the $\left\langle B_{z}\right\rangle$ values are probably overestimated by a significant amount, perhaps by as much as $15-$ $20 \%$. This is supported by the minimum value of $\chi^{2} / v=0.71$, when we expect a value considerably closer to 1.0 .
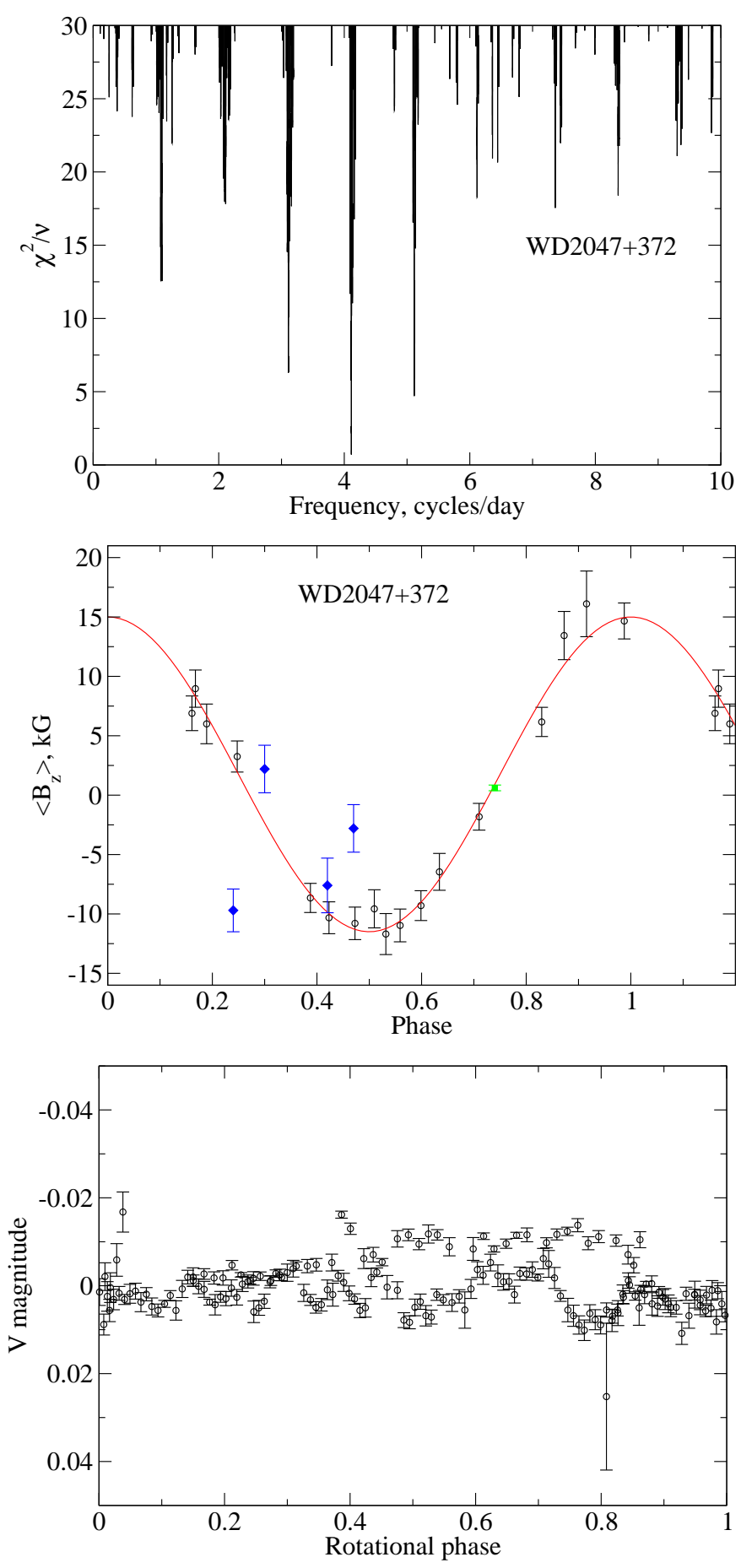

Fig. 3. WD 2047+372. Top panel: chi-squared spectrum sine wave fits to all ESPaDOnS $\left\langle B_{z}\right\rangle$ measurements as a function of frequency of field variation. Mid panel: variation of $\left\langle B_{z}\right\rangle$ as a function of phase through Eq. (5), with the origin of phase at $\left\langle B_{z}\right\rangle$ maximum (positive) $\left\langle B_{z}\right\rangle$. The very precise measurement at phase 0.74 (green point) is the first $\left\langle B_{z}\right\rangle$ value measured using ISIS. The four data points with blue filled diamonds are the MSS data. The smooth curve is the best sine wave fit to the ESPaDOnS $\left\langle B_{z}\right\rangle$ measurements found from the chi-squared period search. Bottom panel: $m_{V}$-band flux variations using the same ephemeris as above.

Because the $\left\langle B_{z}\right\rangle$ value from the single ISIS measurement was determined in quite a different manner from those derived from ESPaDOnS measurements, and may measure the field in a somewhat different way than is done with the ESPaDOnS data, 
the ISIS $\left\langle B_{z}\right\rangle$ value was not used in determining the rotation period of WD 2047+372. However, we have plotted the ISIS $\left\langle B_{z}\right\rangle$ value in Fig. 3, where it appears as a very precise point at about phase 0.74 . The ISIS measurement is in fact in very good agreement with the ensemble of ESPaDOnS data (discussed below), and the fact that it did not reveal a value of $\left\langle B_{z}\right\rangle$ that is significantly different from zero, in spite of very high precision, is simply due to the moment chosen for observation.

The four $\left\langle B_{z}\right\rangle$ measurements obtained with MSS have nominal uncertainties similar to those obtained with ESPaDOnS. The first and fourth MSS measurements are in reasonable agreement with ESPaDOnS measurements; the second one has the correct sign but is too small in absolute value. The third is seriously inconsistent with the variation of Fig. 3 (mid panel), as it deviates from the curve by about $6 \sigma$. The discordant results may result from strongly variable seeing and transparency conditions during the observations, together with total integrations times that are about 0.15 cycles long, leading to errors in the estimated phase of each measurement of 0.05 cycles or more. In any case, the four data points, taken together provide confirmation of a field of approximately $-4.6 \pm 1 \mathrm{kG}$ at about phase 0.36 , consistent with the ESPaDOnS results.

The SAO photometric data were also searched for periodicity. Because the amplitude of variation is comparable to the measurement uncertainties, a number of acceptable frequencies were found. The period deduced from ESPaDOnS $\left\langle B_{z}\right\rangle$ values was one of these, but not the best. The results of the photometric observations phased with the ephemeris of Eq. (5) are presented in the lower panel of Fig. 3, averaged within phase bins of about 0.01 in width. The observational uncertainty $\sigma$ is the standard error of the mean. It is not clear from the figure whether the small variations (of order $0.01 \mathrm{mag}$ ) are actually coherent and reflect real stellar photometric variability, or whether they show the difficulties of trying to detect very small photometric variations under less-than-perfect photometric conditions. In any case, these data appear to establish that any photometric variability of WD $2047+372$ is at most about 0.02 mag peak to peak. This is consistent with the very small magnitude of photometric variations detected for WD 2359-434, and contrasts to the considerably larger photometric variations (up to $\sim 0.1 \mathrm{mag}$ ) observed in some MWDs with much larger magnetic fields (Brinkworth et al. 2013).

\subsection{The rotation period of WD 2359-434}

While the two archival I spectra of WD2359-434 from the UVES archive data are almost identical, and suggest little variability, $\left\langle B_{z}\right\rangle$ measurements from the FORS1 archive data and from our new FORS2 survey are consistent with some rather modest variations of WD 2359-434. The low-resolution FORS spectra yield relatively precise values of $\left\langle B_{z}\right\rangle$, and indicate that $\left\langle B_{z}\right\rangle$ is always positive, and varies between about +2 and $+4 \mathrm{kG}$ (see Table 5). However, these data are too widely spaced in time to allow us to estimate the stellar rotation period. Unfortunately the more closely spaced ESPaDOnS $V / I$ profiles do not even show strong Zeeman polarisation signatures; while almost all of the ESPaDOnS $\left\langle B_{z}\right\rangle$ measurements also report positive fields, these measurements have uncertainties typically five times larger than the FORS data, mostly detect a non-zero value of $\left\langle B_{z}\right\rangle$ only at the $2-3 \sigma$ level, and do not reveal convincing evidence of variations. These data are also not at all well-suited to period finding.

In contrast, the ESPaDOnS intensity spectra do show clear variability, with quite a different pattern of variations compared to those of WD 2047+372, particularly in the shape of the broad $\sigma$ components of the Zeeman pattern, and the overall depth of the line core. The obvious variability of the core of $\mathrm{H} \alpha$ provides the most promising data for searching for periodicity.

The equivalent width data for the continuous series of spectra between MJD 57603 and 57613 were searched for good sine wave fits for periods between 10 and $0.08 \mathrm{~d}$ using DCHI.F. The best fit was found for the period $P=0.11232 \pm 0.00009 \mathrm{~d}$, equivalent to a rotation frequency of $f=8.9031 \pm 0.0071 \mathrm{cycles} / \mathrm{d}$. The time series could have had significant aliasing problems because all observations had to be taken (due to the large southern declination) within a period of about $3 \mathrm{~h}$ each night, but the spread in the local sidereal time of observation from night to night was large enough to clearly identify a unique best period. The aliases with frequency differing from the best frequency by \pm 1 cycle/day are clearly substantially worse. Part of the $\chi^{2} / v$ spectrum for the nine contiguous spectra is shown in Fig. 4

After identifying this period we found the results of Gary et al. (2013), reporting the work of an amateur photometry group which discovered and studied photometric variations of the light from WD 2359-434, as described in Sect. 3.5. They have carried out a time series analysis of their data, and find low-amplitude sinusoidal variation of the light. Based on observations in 2011 and 2012, Gary et al. (2013) propose a period of $P=2.695022 \pm 0.000014 \mathrm{~h}=0.11229258 \pm 0.00000058 \mathrm{~d}$, or $f=8.905308 \pm 0.000046$ cycle/d. Because they have obtained a large number of light curves, many with several full cycles per night, this period is unique. Since the publication of their paper, Gary et al. have obtained further light curves in 2013, 2014, and 2016, and have confirmed and refined somewhat their period, as described on their website ${ }^{4}$. Their period is fully consistent with, but considerably more accurate than, the period derived from the 2016 spectropolarimetry of WD 2359-434.

We then returned to the equivalent width data set, and included the four equivalent width measurements from 2015 in our period search, together with the nine from 2016. Because our data do not uniquely specify the cycle count between OctoberNovember 2015 and August 2016, the allowed frequencies form a comb of acceptable values, of which the best fits are 8.8946, $8.8981,8.9017,8.9052$, and 8.9087 cycles/d. Each of these frequencies is uncertain by about \pm 0.0002 cycles/d. The frequency in best agreement with the results from the photometry of Gary's group is $f=8.9052 \pm 0.0002$ cycles $/ \mathrm{d}(P=0.1122939 \pm$ $0.0000025 \mathrm{~d})$. The two periods are identical within their uncertainties, and it is quite clear that both variable quantities identify the same rotation period. We adopt the period emerging from the consistent results of photometry and spectropolarimetry:

$\operatorname{MJD}\left(\min W_{\lambda}\right)=57324.321+(0.1122926 \pm 0.0000025) \times E,(6)$

where the zero point is chosen as the minimum of the equivalent width (which approximately coincides with the maximum value of $\langle|B|\rangle)$.

The variation of the $\mathrm{H} \alpha$ equivalent width with this period is shown in Fig. 4. Within the uncertainties the variation appears close to sinusoidal.

It is clear from Fig. 1 that, in most ESPaDOnS spectra, a non-zero signal in the $V$ spectrum is barely detectable, and this is reflected in the large relative uncertainties in Table 5. However, the magnitude of $\left\langle B_{z}\right\rangle$ as measured from ESPaDOnS data seems to be about twice as large as the values from FORS. This kind of discrepancy, between values of the mean longitudinal field as measured for a single star by two different techniques, is also found among upper main sequence magnetic stars

4 http://brucegary.net/WDE/WD2359-434/WD2359-434.htm 

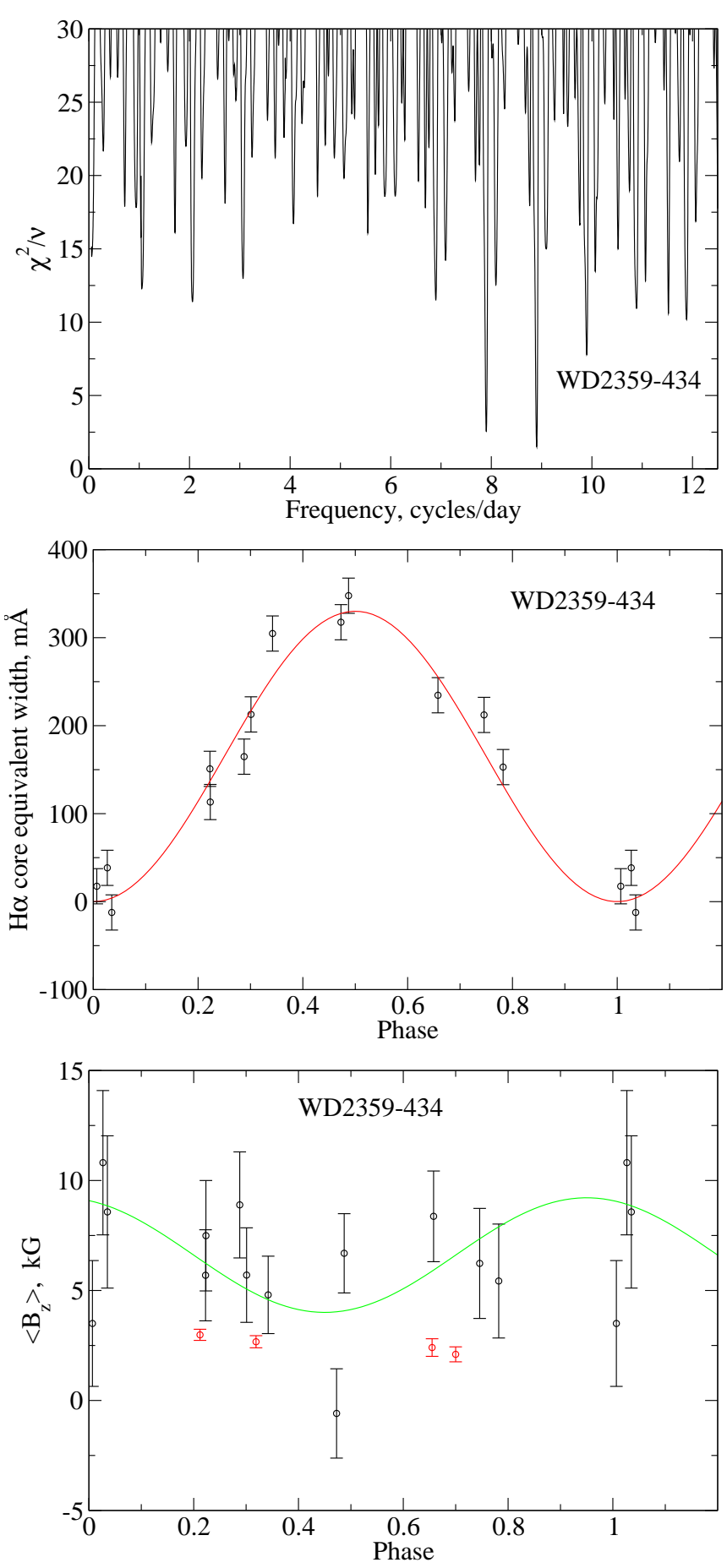

Fig. 4. WD 2359-434. Top panel: chi-squared spectrum of $W_{\lambda}$ measurements of nine contiguous ESPaDOnS spectra of WD 2359-434 taken during ten nights. Mid panel: phased variation of $\mathrm{H} \alpha$ line core equivalent width of WD 2359-434 using the same ephemeris as in Eq. (6). The smooth curve is the best fit sine wave. Bottom panel: variation of $\left\langle B_{z}\right\rangle$ of WD 2359-434 measured from ESPaDOnS spectra (black symbols) and from FORS spectra (red symbols), using the same ephemeris as above. The first two FORS1 measurements are not shown because they are too old to be accurately phased with the ephemeris. The smooth curve is the best fit sine wave to the ESPaDOnS data.

(Borra \& Landstreet 1980; Mathys 1991). In this case, the difference in the scale of $\left\langle B_{z}\right\rangle$ measurements may arise because a method that uses the core of $\mathrm{H} \alpha$ and a method that uses the wings of higher members of the Balmer series will certainly have different limb darkening and line weakening behaviour as a function of colatitude from the centre of the stellar disk, so that in the two kinds of measurement, various parts of the visible disk are given different weights in the computation of the observed values of $\left\langle B_{z}\right\rangle$.

\section{Modelling of the surface magnetic field structure}

In this section we present simple magnetic models that reproduce the field moment measurements (the mean field modulus and mean longitudinal magnetic field) of WD 2047+372 and WD 2359-434. We experimented with three surface field multipolar expansions of increasing complexity, (1) a pure dipolar field; (2) a multipolar expansion with three axisymmetric, colinear components: dipole, quadrupole, and octupole; and (3) a multipolar expansion with a dipole and an independently orientated non-linear quadrupole. For each of these three models it is straight-forward to compute the distribution over the WD surface of the magnetic field vector, and from the distribution to predict the observed field moments $\langle|B|\rangle$ and $\left\langle B_{z}\right\rangle$.

The simple tilted (oblique) dipolar field has been the simplest model adopted in many earlier studies of Ap/Bp stars, starting with Stibbs (1950). It is essentially characterised by a dipolar field strength at the pole $B_{\mathrm{d}}$ and an angle $\beta$ between the rotation axis and the dipolar axis. The tilt angle between the stellar rotation axis and the line of sight is denoted by $i$.

The axisymmetric combination of a colinear dipole, linear quadrupole and linear octupole has already been used by the fitting programs described by Landstreet \& Mathys (2000). In addition to the angles $i$ and $\beta$ and to the dipolar field strength $B_{\mathrm{d}}$, the additional model parameters are the quadrupole and octupole strength $B_{\mathrm{q}}$, and $B_{\mathrm{o}}$, respectively. This parametrisation is rather similar to the one used by Külebi et al. (2009) to model single-snapshot intensity spectra of magnetic white dwarfs detected in the SDSS data set. Their basic model, a dipole either centred in the star (as all our models are) or decentred along the dipole axis, is quite similar in global surface field structure to the sum of an aligned linear dipole and quadrupole, with their decentring parameter playing roughly the same role as the ratio of $B_{\mathrm{q}} / B_{\mathrm{d}}$ in the multipole expansion (as analytically demonstrated by Deridder et al. 1979). Such models can roughly describe topologically dipolar fields, and can also describe a large area of roughly constant local field $|B|$ around one pole, with a flux concentration (a "magnetic spot") at the other pole. The addition of a colinear octupole basically allows us to modify (e.g. reduce) the large pole-to-equator field strength difference of the pure dipole without introducing a spot, which may be required for mapping based on both $I$ and $V$ spectra phased around a full WD rotation cycle, rather than $I$ only from a single snapshot.

The non-axisymmetric model includes the contribution of a dipole and a non-linear quadrupole, as described by Bagnulo et al. (1996) and Landolfi et al. (1998), and the fitting algorithm is fully described in Bagnulo et al. (2000). This model has three more free parameters than the previous model, because even though it does not include an octupolar component, the orientation of the quadrupole is defined by four independent angles, $\left(\beta_{1}, \gamma_{1}\right)$ and $\left(\beta_{2}, \gamma_{2}\right)$.

\section{1. $W D 2047+372$}

Finding a model of WD 2047+372 consistent with our measurements is relatively straightforward. A simple dipolar model with $i=27.0^{\circ} \pm 1.5^{\circ}, \beta=86.5^{\circ} \pm 1^{\circ}$ and $B_{\mathrm{d}}=91.8 \pm 0.8 \mathrm{kG}$ reproduces the $\left\langle B_{z}\right\rangle$ and $\langle|B|\rangle$ observations with a reduced $\chi^{2} \sim 0.8$ 

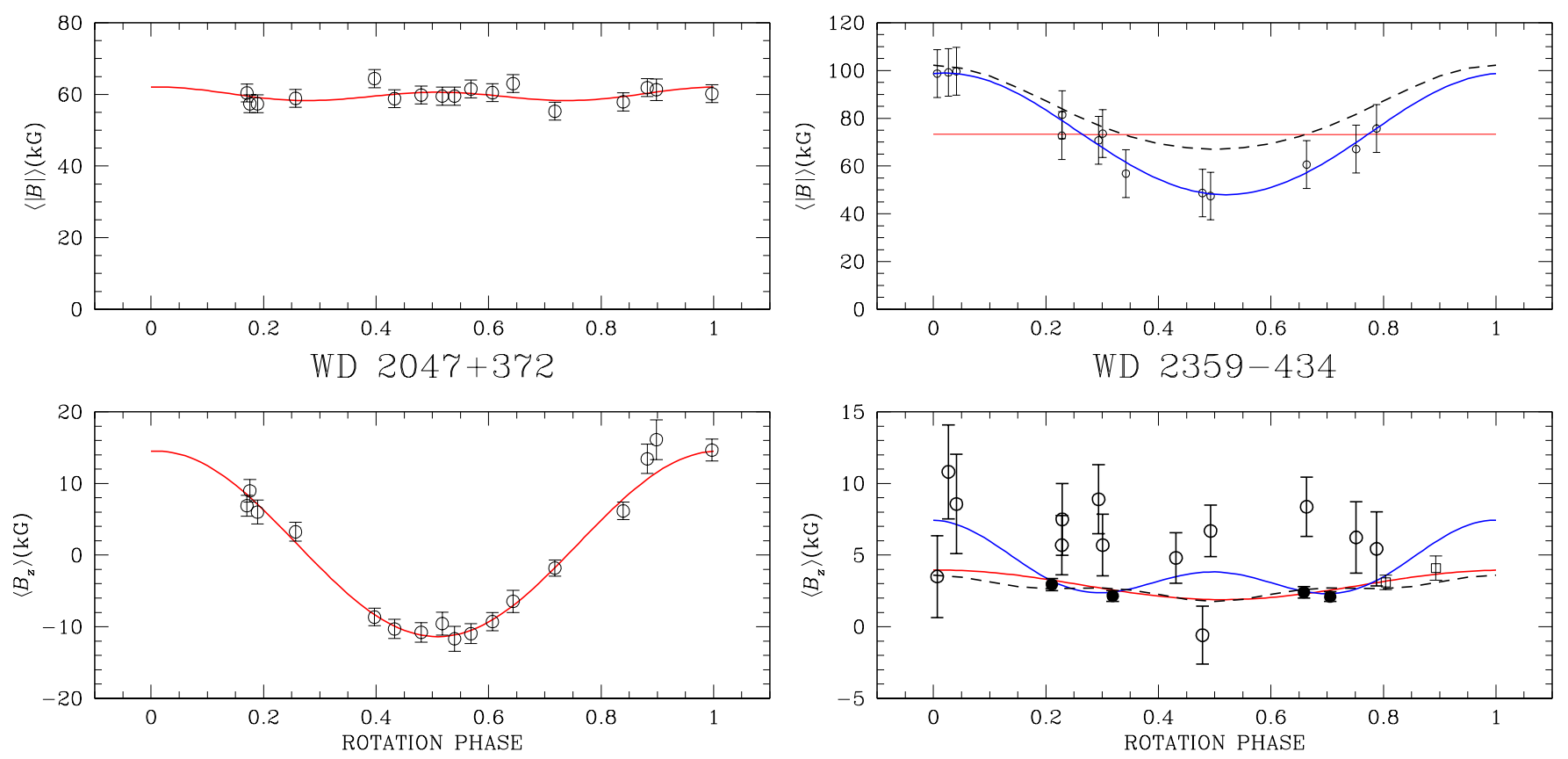

Fig. 5. Left panel: WD 2047+372: observed variation of $\left\langle B_{z}\right\rangle$ and $\langle|B|\rangle$ fit by a simple dipolar model. Right panel: WD 2359-434: observed variation of $\left\langle B_{z}\right\rangle$ and $\langle|B|\rangle$ fit by a simple dipolar model (thin red lines), a co-linear dipole-quadrupole-octupole model (dashed black lines), and by a model including the superposition of a dipole with a non linear quadrupole (thick solid blue lines), as described in the text. Both FORS data (solid circles) and ESPaDOnS data (empty circles) have been used in the fits, with appropriate weights. The two oldest FORS1 data points are shown with nominal (but very uncertain) phases with open squares, but were not used in finding any of the models.

(see left panels of Fig. 5). The $\chi^{2}$ value of just less than 1.0 suggests that our error bars are slightly overestimated. Within the framework of a simple dipolar model, the solution is unique, although it is not possible to distinguish the degenerate solutions in which the $(i, \beta)$ values are exchanged as follows: $(i, \beta),(\beta, i)$, $\left(180^{\circ}-i, 180^{\circ}-\beta\right),\left(180^{\circ}-\beta, 180^{\circ}-i\right)$ (see Landolfi et al. 1998). The parameter values are mildly sensitive to the assumed behaviour of the model stars, particularly the assumed limb darkening and line weakening towards the limb, but changing the assumptions about these effects has only a small effect on the resulting model.

It is found that an axisymmetric model with a mix of dipole and (opposing) octupole fits the moment data as well as the simple dipole. A successful model of this type has $B_{\mathrm{d}}=80 \mathrm{kG}$, $B_{\mathrm{o}}=-40 \mathrm{kG}$, and $(i, \beta) \simeq\left(85^{\circ}, 33^{\circ}\right)$. In comparison with the simple dipole model, which has a polar field strength of $92 \mathrm{kG}$, declining to about $46 \mathrm{kG}$ at the equator, the dipole-octupole field has a polar field of $40 \mathrm{kG}$, rising to about $80 \mathrm{kG}$ around the equator. This difference makes it clear that the moment data do not strongly constrain possible modest variations of local field strength from pole to equator to opposite pole. Comparison of the two field models also shows that using the (net) polar field strength as a measure of the typical MWD field is not nearly as robust as using actual values of $\langle|B|\rangle$ if these are measurable.

\subsection{WD $2359-434$}

For WD 2359-434 the situation is more complicated. The longitudinal field $\left\langle B_{z}\right\rangle$ as measured using FORS appears to be systematically smaller (by about a factor of two) compared to the values measured with ESPaDOnS. Furthermore, although it is clear that $\langle|B|\rangle$ is variable with rotation phase, and that its order of magnitude of $\langle|B|\rangle$ is $\sim 50-100 \mathrm{kG}$, its estimate is not very accurate. A third peculiarity is the small ratio of $\left\langle B_{z}\right\rangle /\langle|B|\rangle$, which is clearly below about 0.1 at all phases in the FORS set of our $\left\langle B_{z}\right\rangle$ measurements. Within the framework of a dipolar model (or any axisymmetric model) this feature can be described by a magnetic field characterised by a strong field at the pole, with the magnetic axis always nearly perpendicular to the line of sight as the star rotates, so that the longitudinal field, averaged over the stellar disk, is very low compared to the mean field modulus. This could be obtained either by $(i, \beta) \simeq\left(0^{\circ}, 90^{\circ}\right)$ or $(i, \beta) \simeq\left(90^{\circ}, 0^{\circ}\right)$. Because the star shows rather strong spectroscopic variability, it is unlikely that the rotation axis is seen pole-on, therefore our favourite solution is $(i, \beta) \simeq\left(90^{\circ}, 0^{\circ}\right)$. The best-fit obtained with a pure dipolar model is shown with red lines in the right panels of Fig. 5, and is obtained with $(i, \beta) \sim\left(87^{\circ}, 1.5^{\circ}\right)$, and a dipolar strength $B_{\mathrm{p}} \sim 145 \mathrm{kG}$. Clearly this model cannot account for the large changes in $\langle|B|\rangle$ that are observed as the star rotates.

A co-linear dipole-quadrupole-octupole model can be found that more closely reproduces the observed weakness of $\left\langle B_{z}\right\rangle$ and the large and variable $\langle|B|\rangle$ field, by making use of the fact that both quadrupole and octupole also produce weakly non-zero $\left\langle B_{z}\right\rangle$ values. It is found that a model with $i=60^{\circ}, \beta=40^{\circ}$, $B_{\mathrm{d}}=+20 \mathrm{kG}$ and $B_{\mathrm{q}}=B_{\mathrm{o}}=-100 \mathrm{kG}$ has a closer resemblance to the observed field moment variations than a simple dipole. The field moments predicted by this model are shown in the right panels of Fig. 5 as black dashed lines.

A much better model that also accounts for the observed variability of the mean field modulus is obtained by considering the superposition of a dipole and a non-axisymmetric quadrupole, and is shown in the right panels of Fig. 5 with blue thick solid lines. This model has the rotation axis inclined to the line of sight by $i=83^{\circ}$, a dipolar field $(\sim 107 \mathrm{kG})$ tilted at $2^{\circ}$ with respect to the rotation axis, and a $63 \mathrm{kG}$ quadrupolar component whose orientation is defined by the angles $\left(\beta_{1}, \gamma_{1}\right)=\left(43^{\circ}\right.$, $\left.14^{\circ}\right)$, and $\left(\beta_{2}, \gamma_{2}\right)=\left(71^{\circ}, 342^{\circ}\right)$. However, the non-axisymmetric model is not unique, so this model should be considered as a 

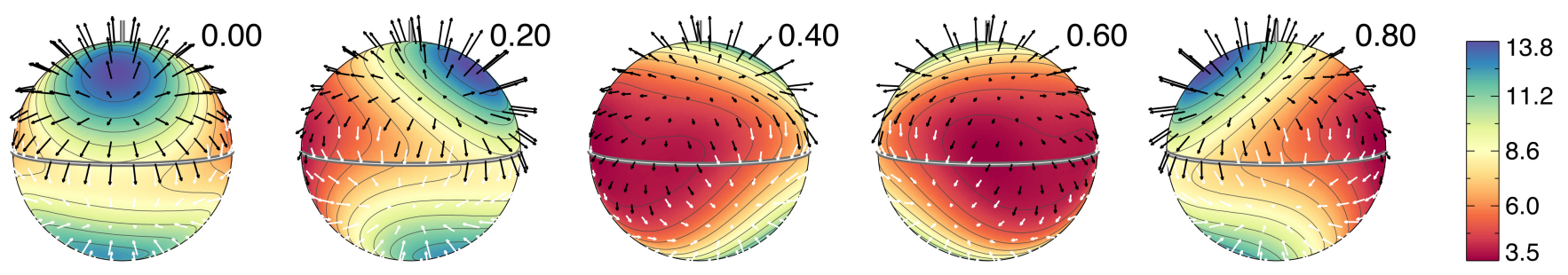

Fig. 6. The distribution of magnetic field over the surface the dipole-non-aligned quadrupole model of WD 2359-434, as seen at five successive phases (left to right: phases 0.0, 0.2, 0.4, 0.6 and 0.8). Black arrows represent outward field, white arrows inward field. The axis of rotation is a small white line segment close to the top of each sphere. The scale at right is in units of $10 \mathrm{kG}$ (e.g. $13.8=138 \mathrm{kG})$.

reasonable example of a simple multipolar field that reproduces the field moment data. The distribution of $|B|$ over the surface of this model is illustrated in Fig. 6.

\section{Spectral synthesis}

Clearly our simple models of WD 2047+372 and WD 2359-434, found by fitting only $\left\langle B_{z}\right\rangle$ and $\langle|B|\rangle$ variations, are expected to be at best rather simplified approximations to the large-scale structures of the real surface field distributions. They are also not unique except within strictly limited families of models. However, in principle we have further information about the field surface geometry in the actual observed $I$ and $V$ line profiles from which the field moments are derived. These original profiles could be compared with line profiles calculated on the basis of the model. Such comparisons could represent useful tests of whether or not our models are usefully realistic. Furthermore, discrepancies between observed and computed $I$ and $V$ line profiles can be used to improve the model surface field distributions.

With a code for spectral synthesis of the magnetic atmospheres of degenerate stars, one could think of performing modelling by inverting the observed Stokes profiles, as is done for instance to map the magnetic field geometries of a number of main sequence magnetic Ap/Bp stars (e.g. Kochukhov et al. 2011; Silvester et al. 2015), and also to model global field geometry of some strong-field WDs (e.g. Jordan 1992; Euchner et al. 2005; Külebi et al. 2009). However, such a project encounters a number of specific problems: MWDs are much fainter than the main sequence stars so far studied, so the time resolution and $\mathrm{S} / \mathrm{N}$ of the data are generally lower; only a few spectral lines are present in the entire optical spectrum; and for most known MWDs no polarimetric data at all are available. Finally, mapping of main sequence magnetic stars is greatly aided by the fact that most show significant rotational line broadening, allowing different parts of the observed line profile to be associated with different locations on the projected visible disk of the star. This is generally not possible for MWDs, whose line cores are not significantly broadened by rotation, but are broadened by pressure (to a full width of more than $1 \AA$ ).

Furthermore, computing a model of the observed line profiles faces a number of important obstacles. A major set of problems concerns the $\mathrm{H} \alpha$ line cores on which we have relied for much of the data discussed in this paper. This line core is strongly affected by departure from LTE, and is not modelled adequately by LTE codes: computed LTE cores are much shallower than those observed (Greenstein \& Peterson 1973; Koester \& Herrero 1988). Furthermore, the correct computation of even the inner line wings requires specification of both absorptive and dichroic line coefficients (Beckers 1969; Wittmann 1974). In addition, even for the relatively weak fields of the

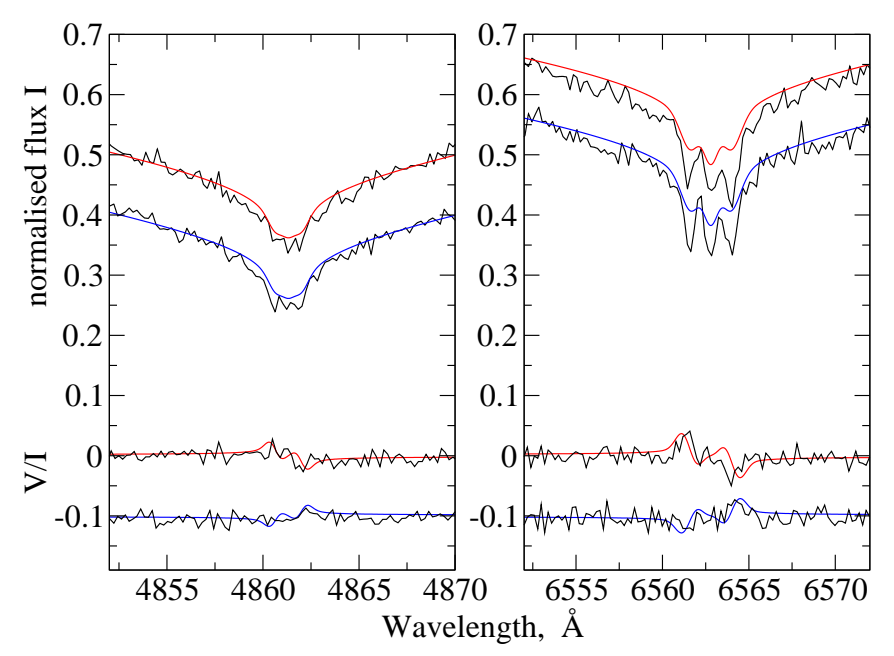

Fig. 7. Line synthesis of $\mathrm{H} \beta$ (left panel) and $\mathrm{H} \alpha$ (right panel) for WD $2047+372$. Observed black $I$ and $V$ curves paired with red models are correctly normalised, and lower curves paired with blue models have been shifted downward by 0.1 for clarity. Upper and lower spectra are from phases $0.98\left(\left\langle B_{z}\right\rangle\right.$ maximum) and $0.53\left(\left\langle B_{z}\right\rangle\right.$ minimum) respectively.

MWDs of interest here, the actual form of the Stark broadening of the $\mathrm{H}$ lines in the presence of a magnetic field is unknown; we must use rough approximations (Jordan 1992; Friedrich et al. 1994). Nevertheless, we have carried out some experimental computations of the line cores of $\mathrm{H} \alpha$ and $\mathrm{H} \beta$ using the code ZEEMAN.F (Landstreet 1988) to see whether these can help us to test and improve the models.

For synthesis of $\mathrm{H} \alpha$ and $\mathrm{H} \beta$ in WD 2047+372, we have simply adjusted the Lorentz damping constants of the line in such a way as to approximately fit the wings close to the line core. Synthesis of $\mathrm{H} \alpha$ using the simple dipolar model of Sect. 6.1 produces a line core that is considerably shallower than the observed core, but has the right qualitative width and shape. For $\mathrm{H} \beta$, which does not have a deep non-LTE core, the computed profile provides an acceptable fit to both the depth and shape of the observed profile. The computed amplitude and shape of the $V$ signal is also compatible with the (low $\mathrm{S} / \mathrm{N}$ ) observed $V$ signature. Comparison of $I$ and $V$ data with synthetic spectra for two representative phases are shown in Fig. 7. Using the dipole-octupole model mentioned in the previous section only marginally changes the computed line profiles, as the changes in the local field strengths and displacements of the $\sigma$ components are small compared to the pressure-broadened line-core width. Thus all the data suggest that the magnetic field of WD 2047+372 may well be axisymmetric, with a local field strength that does not vary around a mean of about $60 \mathrm{kG}$ by more than perhaps \pm 30 or $40 \%$, but we have no constraints on local field variations within these limits. 
Synthesis of Balmer lines in WDs of $T_{\text {eff }} \lesssim 10000 \mathrm{~K}$ with our code is currently very unsatisfactory; the deep pointed cores of $\mathrm{H} \alpha$ and $\mathrm{H} \beta$ in non-magnetic WDs are not reproduced even approximately by our computations. In spite of this, synthesis does generate the relatively shallow line cores of WD 2359-434 fairly successfully. However, in order to test the models against the subtle shape changes found in the $\mathrm{H} \alpha$ I spectra of WD 2359-434, we need to be able to compute more believable $\mathrm{H} \alpha$ line profiles than we are able to at present. This is unfortunate, as the varying shape of the $\mathrm{H} \alpha$ line cores clearly provides more information about the field than is captured by the field moments. In particular, the great breadth of the Zeeman $\sigma$ components in WD 2359434 tells us that the dispersion of the local field strength over the visible hemisphere is very much larger, especially around phases of $\langle|B|\rangle$ maximum, than is the case for WD $2047+372$. This dispersion suggests that there may also be a substantial amount of $V$ signal cancellation over the observed hemisphere, leading to the different values of $\left\langle B_{z}\right\rangle$ deduced from FORS and ESPaDOnS observations.

\section{Discussion and conclusions}

We have reported detection of obvious magnetic variability in the cores of $\mathrm{H} \alpha$ of two weak field magnetic white dwarfs: WD 2047+372 and WD 2359-434. For WD 2047+372, the shape of the Zeeman split line core is almost constant, but polarimetry reveals that the line of sight component of the field, $\left\langle B_{z}\right\rangle$, varies strongly. In contrast, for WD 2359-434, the polarimetric signal is weak and varies relatively little, but the line profile in intensity reveals large changes in the shape of the Zeeman $\sigma$ components, reflecting strong changes in the field strength distribution on opposite sides of this star.

For each of the stars, the measurements reveal a unique stellar rotation period. The rotation period of WD 2047+372, $0.24317 \mathrm{~d}$, is detected for the first time in our data. Furthermore, the internal consistency of the $\left\langle B_{z}\right\rangle$ measurements used to determine the period strongly confirms the stability and precision of the ESPaDOnS spectra (and even suggests that our assigned standard errors of measurement are somewhat too conservative). For this star we report new photometry, which does not show clear periodic variation with the rotation period, but does limit any variations to a semi-amplitude of about $0.01 \mathrm{mag}$.

The newly discovered magnetic variability of WD 2359-434 leads to a rotation period of $0.112292 \mathrm{~d}$, a period found independently from our spectra and from photometry, from which it is found that recent light minima of WD 2359-434 occurred on MJD 57631.785 (phase 0.09 of our ephemeris), and on MJD 57635.833 (phase 0.14); the small phase difference probably arises from the uncertainties of fitting sine waves to data strings of individual nights. It thus appears that minimum light occurs near or shortly after the closest approach of the line of sight to the region of largest field strength, while maximum light occurs about the time when the line of sight intersects the region of relatively weak field.

We have used the new data to obtain simple models of the magnetic field configurations of both the MWDs studied here.

The variations with rotational phase of $\left\langle B_{z}\right\rangle$ and $\langle|B|\rangle$ observed for WD 2047+372 suggest a field structure that is globally dipolar in structure. It appears that the field axis is inclined to the rotation axis by $\sim 30^{\circ}$, and that the rotation axis is inclined almost normal to the line of sight, at about $i=87^{\circ}$ (or, alternatively, $i \sim 30^{\circ}$ and $\beta=87^{\circ}$ ). The local field strength $|B|$ appears to be roughly constant over the surface at about $60 \mathrm{kG}$. This model appears to be consistent with all available field moment data, and leads to synthesised $\mathrm{H} \alpha$ and $\mathrm{H} \beta I$ and $V$ line profiles that closely resemble observed ones. We are led to conclude that this model provides a plausible low-resolution map of the surface field of WD 2047+372, the first such map derived for a super-weak field MWD (this field structure, with rather uniform $\langle|B|\rangle$, is remiscent of the very uniform value deduced for the $200 \mathrm{MG}$ field of HE 0241-0155 by Reimers et al. 2004).

The H $\alpha$ line core of WD 2359-434 shows clear Zeeman splitting, with strongly variable width and depth, indicating the presence of a strongly variable field of the order of $50-100 \mathrm{kG}$. We have found that the magnetic field of WD 2359-434 is very probably not axisymmetric, and we have identified a simple multipolar model that reproduces the variations of $\left\langle B_{z}\right\rangle$ and $\langle|B|\rangle$. Our $\mathrm{H} \alpha$ line profiles clearly contain more information than is captured by the field moments. However, modelling of the actual $\mathrm{H} \alpha$ line cores of this star to test and refine possible field models still presents major challenges.

Nevertheless, it is clear that the magnetic field of WD 2359-434 does not resemble that of a dipole centred in the star, and in fact does not even have an axis of symmetry. The field shows very large variation over the surface (a factor of order three or more), especially when the stronger field region is visible. This field structure, although not uniquely modelled, is clearly very different from that of WD 2047+372, on which the local field does not appear to vary much in strength over the visible surface.

The origin of the major differences between these two surface field geometries is not clear at present. In fact, starting from the view that the fields of these two stars are fossil fields, one would expect that the complexity of the global field structure would decrease with increasing age, as the higher-order multipole components decay ohmically more rapidly than lowerorder terms (Cowling 1976, Sect. 1.3). In this case, if the two stars started their lives with fields of comparable complexity, we would expect WD 2359-434, which is four times older than WD 2047+372, to have the simpler field, or perhaps even a roughly dipolar field. Instead, we find the exact opposite situation: WD 2359-434 has a much more complex surface field than WD 2047+372, whose field shows no indication of significant departure from a simple dipole.

We note that the field structure modelling carried out here is unusual in that it is based on spectropolarimetry carried out throughout known rotation periods. Although there has been a lot of modelling of spectroscopy and spectropolarimetry of MWDs to obtain information about the surface magnetic field distributions, this has generally been based only on one or a few snapshot observations. Models based on observations spread through a rotation cycle, which are considerably better constrained than those based on snapshots, have been published for only a few MWDs, including WD 0009+501 (Valyavin et al. 2005, 2006; Valeev et al. 2015), Feige 7 (Achilleos et al. 1992), RE J0317-853 (Burleigh et al. 1999), PG 1015+014 (Euchner et al. 2006), PG 1031+234 (Schmidt et al. 1986), HE 1045-0908 (Euchner et al. 2005), and WD 1953-011 (Maxted et al. 2000; Valyavin et al. 2008). With the exception of WD 0009+501, all of these MWDs have fields of $10 \mathrm{MG}$ or more. Our models are the first based on full-phase data for any extremely weak-field MWDs.

It is of interest to consider the two MWDs studied here in the context of the seven MWDs known to have fields $\langle|B|\rangle$ below about $200 \mathrm{kG}$ (the MWDs closest to the threshold of field detection), which are summarised in Table 6 . Until recently, little more was known about the fields of these stars beyond a snapshot value for each star of $\langle|B|\rangle$ or $\left\langle B_{z}\right\rangle$. With this paper 
Table 6. Confirmed MWDs with magnetic fields of less than about $200 \mathrm{kG}$.

\begin{tabular}{cccccccc}
\hline \hline WD designation & Name & $\begin{array}{c}m_{V} \\
(\mathrm{mag})\end{array}$ & $\begin{array}{c}\text { Spectral } \\
\text { class }\end{array}$ & $\begin{array}{c}T_{\text {eff }} \\
(\mathrm{K})\end{array}$ & $\begin{array}{c}\text { Typical }\langle|B|\rangle \\
(\mathrm{kG})\end{array}$ & $\begin{array}{c}\text { Period } \\
(\mathrm{days})\end{array}$ & Photometry \\
\hline WD 0446-789 & BPM 3523 & 13.47 & DA 2.1 & 24440 & 15 & $\mathrm{~V}$ & ND \\
WD 2105-820 & LTT 8381 & 13.59 & DA 4.8 & 10600 & 43 & NV & ND \\
WD 2047+372 & LTT 16093 & 13.40 & DA 3.4 & 14710 & 60 & 0.2432 & V? \\
WD 1653+385 & NLTT 43806 & 15.86 & DZA & 5833 & 70 & ND & ND \\
WD 0257+080 & LHS 5064 & 15.90 & DA 7.8 & 6340 & 90 & ND & ND \\
WD 2359-434 & LTT 9857 & 13.05 & DAP 5.8 & 8650 & $50-150$ & 0.1123 & V \\
WD 0322-019 & G77-50 & 16.22 & DAZ & 5195 & 120 & 29.8 or 1.03 & ND \\
\hline
\end{tabular}

Notes. $m_{V}$ and spectral class from Simbad database; $T_{\text {eff }}$ from Gianninas et al. (2011) and Giammichele et al. (2012); typical $\langle|B|\rangle$ for WD 0446789, WD 2047+372, and WD 2359-434 from our own estimates, for WD 2105-820 from Landstreet et al. (2012), for WD 1653+385 from Zuckerman et al. (2011), for WD 0257+080 from Koester et al. (2009), and for WD 0322-019 from Farihi et al. (2011); for period from this work and Farihi et al. (2011). In the period and photometry columns, ND: no data; NV: not variable; V: variable.

we have considerably increased the amount of information about two very different members of this highly interesting set. It will be of great interest to obtain the necessary data for modelling a few more of these stars.

One encouraging aspect of the summary of super-weak field stars is already clear from Table 6, namely that discovery of this field strength range among bright WDs must still be incomplete, as four (more than half) of the stars in Table 6 are brighter than $m_{V}=13.6$. No stars in the magnitude range 13.6 to 15.8 are found in the table, although we would expect at least 8 times as many super-weak MWDs in this magnitude interval as are found brighter than 13.6. Many of these relatively bright but undiscovered super-weak field MWDs must be among the numerous known WDs in this magnitude range. Thus, continued searches with high-resolution instruments are likely to substantially enlarge the relatively bright, easily studied weak-field sample.

The photometric variations found in one of the two superweak MWDs studied here, and our upper limit for the other, are consistent with similar low-amplitude variations observed in two other weak field MWDs, WD 0009+501 (=G217-37) and WD 0853+163 (=LB 8915). However, the amplitudes of the variations of the two stars studied here, less than $1 \%$ peak-topeak amplitude, are somewhat smaller than the variations observed in the two other stars, and considerably smaller than those reported for a few MWDs with MG fields (Brinkworth et al. 2013). The explanation for very low-amplitude light variability in such weakly magnetic WDs is not obvious.

The results presented here clearly demonstrate that a $4 \mathrm{~m}$ class telescope equipped with a medium- or high-resolution, high-efficiency spectrograph (or even better, a spectropolarimeter) can provide data from the core of $\mathrm{H} \alpha$ that reveal aspects of white dwarf magnetism that are not accessible, especially for the weakest fields, with the conventional low-resolution instruments normally used to observe white dwarfs. Because it is not clear in advance whether the $I$ or the $V$ spectrum of a weakfield MWD will prove more useful for studying variability and constraining field models, it is particularly valuable to use a spectropolarimeter.

Acknowledgements. We thank the Queue Service Observing team at the CFHT for outstanding help with interactive scheduling of our observations, and for providing us with long, consistent data sets. We thank Dr. Oleg Kochukhov (Uppsala University) for very helpful advice and a macro for making Fig. 6. J.D.L. acknowledges financial support from the Natural Sciences and Engineering Research Council of Canada. G.V. and A.V. acknowledges the support from the Russian Foundation for Basic Research (RFBR grant N15-02-05183). This research has made use of the VizieR catalogue access tool, CDS, Strasbourg,
France. The original description of the VizieR service was published in A\&AS, $143,23$.

\section{References}

Achilleos, N., Wickramasinghe, D. T., Liebert, J., Saffer, R. A., \& Grauer, A. D. 1992, ApJ, 396, 273

Angel, J. R. P., \& Landstreet, J. D. 1971, ApJ, 164, L15

Appenzeller, I., Fricke, K., Fürtig, W., et al. 1998, The Messenger, 94, 1

Aznar Cuadrado, R., Jordan, S., Napiwotzki, R., et al. 2004, A\&A, 423, 1081

Bagnulo, S., Landi Degl'Innocenti, M., \& Landi Degl'Innocenti, E. 1996, A\&A, 308,115

Bagnulo, S., Landolfi, M., Mathys, G., \& Landi Degl'Innocenti, M. 2000, A\&A, 358,929

Bagnulo, S., Fossati, L., Landstreet, J. D., \& Izzo, C. 2015, A\&A, 583, A115

Beckers, J. M. 1969, Sol. Phys., 9, 372

Borra, E. F., \& Landstreet, J. D. 1980, ApJS, 42, 421

Brinkworth, C. S., Burleigh, M. R., Lawrie, K., Marsh, T. R., \& Knigge, C. 2013, ApJ, 773, 47

Burleigh, M. R., Jordan, S., \& Schweizer, W. 1999, ApJ, 510, L37

Casini, R., \& Landi Degl'Innocenti, E. 1994, A\&A, 291, 668

Chountonov, G. A. 2004, in Magnetic Stars, eds. N. Arkhyz, Y. V. Glagolevskij, I. I. Kudryavtsev, \& I. I. Romanyuk, 286

Cowling, T. G. 1976, Magnetohydrodynamics, Monographs on Astronomical Subjects (Bristol: Adam Hilger)

Deridder, G., van Rensbergen, W., \& Hensberge, H. 1979, A\&A, 77, 286

Donati, J.-F., Semel, M., Carter, B. D., Rees, D. E., \& Collier Cameron, A. 1997, MNRAS, 291, 658

Euchner, F., Reinsch, K., Jordan, S., Beuermann, K., \& Gänsicke, B. T. 2005, A\&A, 442, 651

Euchner, F., Jordan, S., Beuermann, K., Reinsch, K., \& Gänsicke, B. T. 2006, A\&A, 451, 671

Farihi, J., Dufour, P., Napiwotzki, R., \& Koester, D. 2011, MNRAS, 413, 2559 Ferrario, L., de Martino, D., \& Gänsicke, B. T. 2015, Space Sci. Rev., 191, 111 Friedrich, S., Ostreicher, R., Ruder, H., \& Zeller, G. 1994, A\&A, 282, 179

Gary, B. L., Tan, T. G., Curtis, I., Tristram, P. J., \& Fukui, A. 2013, Society for Astronomical Sciences Annual Symposium, 32, 71

Giammichele, N., Bergeron, P., \& Dufour, P. 2012, ApJS, 199, 29

Gianninas, A., Bergeron, P., \& Ruiz, M. T. 2011, ApJ, 743, 138

Greenstein, J. L., \& Liebert, J. W. 1990, ApJ, 360, 662

Greenstein, J. L., \& Peterson, D. M. 1973, A\&A, 25, 29

Jordan, S. 1992, A\&A, 265, 570

Kawka, A., Vennes, S., Schmidt, G. D., Wickramasinghe, D. T., \& Koch, R. 2007, ApJ, 654, 499

Kemp, J. C., Swedlund, J. B., Landstreet, J. D., \& Angel, J. R. P. 1970, ApJ, 161, L77

Kepler, S. O., Pelisoli, I., Jordan, S., et al. 2013, MNRAS, 429, 2934

Kochukhov, O., Lundin, A., Romanyuk, I., \& Kudryavtsev, D. 2011, ApJ, 726, 24

Koester, D., \& Herrero, A. 1988, ApJ, 332, 910

Koester, D., Dreizler, S., Weidemann, V., \& Allard, N. F. 1998, A\&A, 338, 612 Koester, D., Voss, B., Napiwotzki, R., et al. 2009, A\&A, 505, 441

Külebi, B., Jordan, S., Euchner, F., Gänsicke, B. T., \& Hirsch, H. 2009, A\&A, 506, 1341

Landolfi, M., Bagnulo, S., \& Landi Degl'Innocenti, M. 1998, A\&A, 338, 111 
J. D. Landstreet et al.: Short-period magnetic variability of two super-weak field white dwarfs

Landstreet, J. D. 1988, ApJ, 326, 967

Landstreet, J. D., \& Angel, J. R. P. 1971, ApJ, 165, L67

Landstreet, J. D., \& Mathys, G. 2000, A\&A, 359, 213

Landstreet, J. D., Silaj, J., Andretta, V., et al. 2008, A\&A, 481, 465

Landstreet, J. D., Bagnulo, S., Valyavin, G. G., et al. 2012, A\&A, 545, A30

Landstreet, J. D., Bagnulo, S., \& Fossati, L. 2014, A\&A, 572, A113

Landstreet, J. D., Bagnulo, S., Valyavin, G. G., et al. 2015, A\&A, 580, A120

Landstreet, J. D., Bagnulo, S., Martin, A., \& Valyavin, G. 2016, A\&A, 591, A80

Mathys, G. 1989, Fund. Cosmic Phys., 13, 143

Mathys, G. 1991, A\&AS, 89, 121

Maxted, P. F. L., Ferrario, L., Marsh, T. R., \& Wickramasinghe, D. T. 2000, MNRAS, 315, L41

Neiner, C., Landstreet, J. D., Alecian, E., et al. 2012, A\&A, 546, A44

Putney, A., \& Jordan, S. 1995, in White Dwarfs, eds. D. Koester, \& K. Werner (Berlin: Springer Verlag), Lect. Notes Phys., 443, 135

Reimers, D., Jordan, S., \& Christlieb, N. 2004, A\&A, 414, 1105
Schmidt, G. D., \& Smith, P. S. 1995, ApJ, 448, 305

Schmidt, G. D., West, S. C., Liebert, J., Green, R. F., \& Stockman, H. S. 1986, ApJ, 309, 218

Silvester, J., Kochukhov, O., \& Wade, G. A. 2015, MNRAS, 453, 2163

Stibbs, D. W. N. 1950, MNRAS, 110, 395

Valeev, A. F., Antonyuk, K. A., Pit, N. V., et al. 2015, Astrophys. Bull., 70, 318 Valyavin, G. 2015, in Physics and Evolution of Magnetic and Related Stars, eds

Y. Y. Balega, I. I. Romanyuk, \& D. O. Kudryavtsev, ASP Conf. Ser., 494,107 Valyavin, G., Bagnulo, S., Monin, D., et al. 2005, A\&A, 439, 1099

Valyavin, G., Bagnulo, S., Fabrika, S., et al. 2006, ApJ, 648, 559

Valyavin, G., Wade, G. A., Bagnulo, S., et al. 2008, ApJ, 683, 466

Valyavin, G. G., Bychkov, V. D., Yushkin, M. V., et al. 2015, in Physics and Evolution of Magnetic and Related Stars, eds. Y. Y. Balega, I. I. Romanyuk, \& D. O. Kudryavtsev, ASP Conf. Ser., 494, 305

Wittmann, A. 1974, Sol. Phys., 35, 11

Zuckerman, B., Koester, D., Dufour, P., et al. 2011, ApJ, 739, 101 